\title{
Hermite pesudospectral method and modified Hermite spectral method for long-short wave equations
}

\author{
Zeting Liu, Shujuan Lü* \\ School of Mathematics and Systems Science \& LMIB, Beihang University, Beijing 100191, China.
}

Communicated by A. Atangana

\begin{abstract}
We consider the initial boundary value problem of the long-short wave equations on the whole line. Firstly, a fully discrete Hermite pseudospectral scheme and modified Hermite spectral scheme are structured basing Hermite functions, respectively. Secondly, we analyze the two kinds of schemes theoretically. The modified Hermite spectral scheme shows the superiority in priori estimates, numerical stability and convergence. Thirdly, numerical experiments for the two schemes are presented to confirm our theoretical analysis. (C)2017 All rights reserved.
\end{abstract}

Keywords: Long-short wave equations, Hermite pseudospectral method, modified Hermite spectral method, convergence, stability.

2010 MSC: 35B45, 65M12, 76M22.

\section{Introduction}

Many problems in science and engineering are set in unbounded domains. To solve these PDEs numerically, scientists use the finite difference method or the finite element method usually restrict calculations to some bounded domains, and impose certain conditions on artificial boundaries which often cause numerical errors [5]. While spectral method can avoid the troubles mentioned above and can also provide numerical solutions with high accuracy. We can use classical orthogonal systems defined in unbounded domains, for example, using Laguerre spectral methods in semi-unbounded domains or exterior domains $[6,8,10,12,18,20]$ and using Hermite spectral methods in whole unbounded domains $[2,7,11,14,22,24]$. Laguerre and Hermite spectral methods are attractive because of their high accuracy and freedom from artificial boundary conditions.

In this paper, we consider the following long-short wave (LS) equations:

$$
\left\{\begin{array}{l}
\text { is } s_{t}+s_{x x}=\alpha s l+f, \quad x \in \mathbf{R}, 0<t \leqslant T, \\
l_{t}+\beta\left(|s|^{2}\right)_{x}=g, \quad x \in \mathbf{R}, 0<t \leqslant T \\
s(x, 0)=s_{0}(x), \quad l(x, 0)=l_{0}(x), \quad x \in \mathbf{R}, \\
\lim _{|x| \rightarrow \infty} s(x, t)=\lim _{|x| \rightarrow \infty} s_{x}(x, t)=\lim _{|x| \rightarrow \infty} l(x, t)=0, \quad 0<t \leqslant T,
\end{array}\right.
$$

\footnotetext{
*Corresponding author

Email addresses: 1zt_well@163.com (Zeting Liu), 1sj@buaa.edu.cn (Shujuan Lü)
} 
where complex function $s$ is the envelope of the short wave, real function $l$ is the amplitude of the long wave and $\alpha, \beta$ are positive numbers, $f$ and $g$ are source terms (force terms).

We use Hermite functions to approximate the solutions of LS equations. As is known to all Hermite polynomials methods with weight $\omega(x)=e^{-x^{2}}$ which can destroy the crucial conservation properties of equations as well as the symmetry and positive definiteness of bilinear operators, may lead to complication in analysis and implementation and also the weight is not natural for some physical problems. Thus it is more appropriate to consider approximation by Hermite functions with weight $\omega(x)=1$. By using these Hermite functions, we develop Hermite pseudospectral method and the modified Hermite spectral method, respectively. There is no work using these two methods for the LS equations in the unbounded domains so far.

The motivation we develop Hermite pseudospectral method is due to the fact that it is not easy to perform the quadratures in unbounded domains especially for the nonlinear terms. But the analysis for pseudospectral method is difficult, especially for the priori estimates, thus we established the modified Hermite spectral method. Comparing with Hermite spectral method, the modified Hermite spectral method treats the nonlinear terms and source terms with collocation method. Then the modified Hermite spectral method is more preferable in actual calculations by using the Hermite-Gauss integral formula. Comparing to the pseudospectral method, the stiffness matrix is sparse while the the stiffness matrix using pseudospectral method is full. Thus the modified Hermite spectral method combines both the advantages of spectral method and pseudospectral method, it is more efficient to implement in practice than the pseudospectral method.

It is worth mentioning that most researchers establish semi-discrete schemes to solve PDEs, see papers we referred above, while a little construct full-discrete schemes. As everyone knows, the latter are more difficult than the former in theoretical analysis. The existing papers $[3,4,15,16]$ using full-discrete schemes to prove error estimate are also under restrict grid conditions which are not conducive to the realization of the algorithms, so it is urgent to seek the full-discrete scheme under no restrict on grid condition.

In this paper, we apply Hermite functions methods for equations (1.1)-(1.4). We firstly study properties of Hermite-Gauss interpolation and obtain the error of the pseudospectral approximation. Secondly, we establish a two level linear fully discrete Hermite pseudospectral scheme and a three level linear fully discrete modified Hermite spectral scheme. Thirdly, we study the two kinds of schemes. The convergence of pseudospectral scheme is the first difficult point in this paper. For the modified Hermite spectral scheme, a priori estimates are the key point as well as the second difficult point in this paper, the numerical stability and the convergence of the discrete scheme are the third difficult point in this paper.

An outline of this paper is as follows: In Section 2, we commence by reviewing some preliminaries and notations. We also recall the properties of the Hermite-Gauss interpolation and obtain the error estimate of the pseudospectral approximation. In Section 3, we prove the convergence of the Hermite pseudospectral method. In Section 4, we study the modified Hermite spectral method including a priori estimates, unconditional numerical stability and the convergence of the fully discrete scheme. In Section 5 , we present numerical results for the two kinds of schemes. Finally, some conclusions are given in Section 6.

\section{Preliminaries and notations}

Let $\mathrm{L}^{2}(\mathbf{R}), \mathrm{L}^{\infty}(\mathbf{R})$, and $\mathrm{H}^{\mathrm{m}}(\mathbf{R})$ the usual Sobolev spaces equipped with norms $\|\cdot\|,\|\cdot\|_{\infty}$, and $\|\cdot\|_{\mathrm{m}}$, respectively. The inner product of $\mathrm{L}^{2}(\mathbf{R})$ and $\mathrm{H}^{\mathrm{m}}(\mathbf{R})$ are denoted by $(\cdot, \cdot)$ and $(\cdot, \cdot)_{\mathrm{m}}$, respectively. $|\cdot|_{\mathrm{m}}$ denotes the semi-norm of $\mathrm{H}^{\mathrm{m}}(\mathbf{R})$. Throughout this paper $\mathrm{c}$ is a generic positive constant independent of $\mathrm{N}$ and any function.

The Hermite polynomial of degree $l$ denoted by $\mathrm{H}_{l}$ has $l$ real and distinct zeros which are symmetric with respect to the origin. If $d_{l}$ denotes the smallest distance between two consecutive zeros of $H_{l}$, then 
(see [21])

$$
\mathrm{d}_{\mathrm{l}} \geqslant \frac{\pi}{(2 \mathrm{l}+1)^{1 / 2}} \geqslant \sqrt{\frac{3}{\mathrm{l}}}, \quad \forall \mathrm{l} \in \mathbf{N}
$$

The Hermite functions of degree $l$ are defined by

$$
\hat{H}_{l}(x)=\frac{1}{\pi^{\frac{1}{4}} \sqrt{2^{l} l !}} e^{-\frac{x^{2}}{2}} H_{l}(x), \quad l=0,1,2, \ldots
$$

$\hat{H}_{l}(x)$ satisfies the following relation:

$$
\hat{\mathrm{H}}_{l x}(x)=\sqrt{2 \mathrm{l}} \hat{\mathrm{H}}_{l-1}(x)-x \hat{\mathrm{H}}_{\mathrm{l}}(x)=\sqrt{\frac{l}{2}} \hat{\mathrm{H}}_{\mathrm{l}-1}(x)-\sqrt{\frac{l+1}{2}} \hat{\mathrm{H}}_{l+1}(x), \quad l \geqslant 1 .
$$

The functions $\hat{H}_{l}(x)$ are mutually-orthogonal in $L^{2}(\mathbf{R})$, i.e.,

$$
\int_{\mathbf{R}} \hat{\mathrm{H}}_{\mathrm{l}}(x) \hat{\mathrm{H}}_{\mathrm{m}}(x) \mathrm{d} x=\delta_{\mathrm{l}, \mathrm{m}}
$$

where $\delta_{l, m}$ is the Kronecker function. Moreover, we have

$$
\int_{\mathbf{R}} \hat{H}_{l x}(x) \hat{H}_{m x}(x) d x= \begin{cases}-\frac{\sqrt{l(l-1)}}{2}, & m=l-2 \\ l+\frac{1}{2}, & m=l \\ -\frac{\sqrt{(l+1)(l+2)}}{2}, & m=l+2 \\ 0, & \text { otherwise }\end{cases}
$$

For any $v \in \mathrm{L}^{2}(\mathbf{R})$, we may write $v(x)=\sum_{l=0}^{\infty} \hat{v}_{l} \hat{\mathrm{H}}_{\mathrm{l}}(\mathrm{x})$, where

$$
\hat{v}_{l}=\int_{\mathbf{R}} v(x) \hat{H}_{l}(x) d x, \quad l=0,1,2, \cdots,
$$

$\hat{v}_{l}$ are the Hermite coefficients. Let $\mathrm{N}$ be any positive integer and

$$
\mathcal{H}_{N}=\operatorname{span}\left\{\hat{\mathrm{H}}_{0}(\chi), \hat{\mathrm{H}}_{1}(\chi), \ldots, \hat{\mathrm{H}}_{\mathrm{N}}(\chi)\right\} \text {. }
$$

Denote by $\mathrm{P}_{\mathrm{N}}: \mathrm{L}^{2}(\mathcal{R}) \rightarrow \mathcal{H}_{\mathrm{N}}$ the orthogonal projection. It satisfies for any $v \in \mathrm{L}^{2}(\mathbf{R})$,

$$
\left(\mathrm{P}_{\mathrm{N}} v-v, \phi\right)=0, \quad \forall \phi \in \mathcal{H}_{\mathrm{N}} .
$$

For any integer $r \geqslant 0$, we define a normed space as follows,

$$
H_{A}^{r}(\mathbf{R})=\left\{u:\|u\|_{r, A}<\infty\right\},
$$

where

$$
\|v\|_{r, A}=\left(\sum_{k=0}^{r}\left\|\left(x^{2}+1\right)^{\frac{r-k}{2}} \partial_{\chi}^{k} v\right\|^{2}\right)^{\frac{1}{2}} .
$$

For any real $r>0$, we define the space and its norm by function space interpolation.

Now we give two lemmas which are frequently used in the following sections.

Lemma 2.1 ([23]). For any $\varphi \in \mathcal{H}_{N}$ and $r \geqslant 0$, we have

$$
\left\|\partial_{x}^{\mathrm{r}} \varphi\right\| \leqslant c N^{\frac{\mathrm{r}}{2}}\|\varphi\| \text {. }
$$


Lemma 2.2 ([23]). For any $v \in \mathrm{H}_{\mathrm{A}}^{\mathrm{r}}(\mathbf{R})$ and $0 \leqslant \mu \leqslant r$, we have

$$
\left|\mathrm{P}_{\mathrm{N}} \nu-v\right|_{\mu} \leqslant \mathrm{cN}^{\frac{\mu-\mathrm{r}}{2}}\|v\|_{\mathrm{r}, \mathrm{A}} \text {. }
$$

Let $\left\{x_{j}\right\}_{j=0}^{N}$ be the zeros of $H_{N+1}$, and let $\left\{w_{j}\right\}_{j=0}^{N}$ be the corresponding modified Hermite-Gauss weight, namely

$$
w_{j}=\frac{1}{(N+1) \hat{H}_{N}^{2}\left(x_{j}\right)} .
$$

The discrete inner product and the discrete norm are defined by

$$
(u, v)_{N}=\sum_{j=0}^{N} u\left(x_{j}\right) v\left(x_{j}\right) w_{j},\|v\|_{N}=(u, v)_{N}^{\frac{1}{2}} .
$$

For any $\varphi \in \mathcal{H}_{m}, \psi \in \mathcal{H}_{2 \mathrm{~N}+1-\mathrm{m}}$, and any non-negative integer $\mathrm{m} \leqslant 2 \mathrm{~N}+1$, then (see [9]),

$$
(\varphi, \psi)=(\varphi, \psi)_{N} .
$$

For any $v \in \mathrm{C}(\mathbf{R})$, the Hermite-Gauss interpolant $\mathrm{I}_{N} v \in \mathcal{H}_{N}$ is determined by

$$
I_{N} v\left(x_{j}\right)=v\left(x_{j}\right), \quad 0 \leqslant j \leqslant N,
$$

or equivalently,

$$
\left(\mathrm{I}_{\mathrm{N}} v-v, \varphi\right)_{\mathrm{N}}=0, \quad \forall \varphi \in \mathcal{H}_{\mathrm{N}} .
$$

Theorem 2.3. For any $v \in \mathrm{H}_{A}^{\mathrm{r}}(\mathbf{R}), \mathrm{r} \geqslant 1$, and $0 \leqslant \mu \leqslant r$,

$$
\left\|\mathrm{I}_{\mathrm{N}} v-v\right\|_{\mu} \leqslant \mathrm{cN}^{\frac{1}{6}+\frac{\mu-\mathrm{r}}{2}}\|v\|_{\mathrm{r}, A} .
$$

Proof. It is shown in [19] that for any $a<b$,

$$
\sup _{x \in[a, b]}|v(x)| \leqslant \frac{1}{\sqrt{b-a}}\|v\|_{L^{2}(a, b)}+\sqrt{b-a}|v|_{H^{1}(a, b)} .
$$

Taking into account equation (2.1), one can choose $\mathrm{N}+1$ disjoint compact subsets $\mathrm{K}_{0}, \mathrm{~K}_{1}, \ldots, \mathrm{K}_{\mathrm{N}}$ such that $x_{j} \in K_{j}$ and $\left|K_{j}\right|=\sqrt{\frac{3}{N}}$ for $0 \leqslant j \leqslant N$. Then by the definition of discrete inner product, using equation (2.5) and $w_{j} \leqslant \mathrm{cN}^{-\frac{1}{6}}([1$, Theorem 4$])$, we deduce that

$$
\begin{aligned}
\|v\|_{N}^{2}=\sum_{j=0}^{N} v\left(x_{j}\right)^{2} w_{j} & \leqslant c N^{-\frac{1}{6}} \sum_{j=0}^{N} \sup _{x \in \mathrm{K}_{j}} v(x)^{2} \\
& \leqslant c N^{-\frac{1}{6}} \sum_{j=0}^{N}\left(\sqrt{\frac{\mathrm{N}}{3}}\|v\|_{\mathrm{L}^{2}\left(\mathrm{~K}_{j}\right)}^{2}+\sqrt{\frac{3}{N}}|v|_{\mathrm{H}^{1}\left(\mathrm{~K}_{\mathrm{j}}\right)}^{2}\right) \\
& \leqslant c N^{\frac{1}{3}}\|v\|_{\mathrm{L}^{2}(\mathcal{R})}^{2}+\mathrm{cN}^{-\frac{2}{3}}|v|_{\mathrm{H}^{1}(\mathcal{R})}^{2} .
\end{aligned}
$$

That is,

$$
\|v\|_{\mathrm{N}} \leqslant \mathrm{cN}^{\frac{1}{6}}\|v\|+\mathrm{cN}^{-\frac{1}{3}}|v|_{1}
$$

By using Lemmas 2.1, 2.2, equation (2.6), and the fact that $I_{N} P_{N} v=P_{N} v$, we infer that

$$
\begin{aligned}
\left\|\mathrm{I}_{N} v-v\right\|_{\mu} & \leqslant\left\|\mathrm{I}_{N}\left(v-\mathrm{P}_{N} v\right)\right\|_{\mu}+\left\|\mathrm{P}_{N} v-v\right\|_{\mu} \\
& \leqslant c N^{\frac{\mu}{2}}\left\|\mathrm{I}_{N}\left(v-\mathrm{P}_{N} v\right)\right\|+\left\|\mathrm{P}_{N} v-v\right\|_{\mu} \\
& \leqslant c N^{\frac{\mu}{2}}\left(\mathrm{~N}^{\frac{1}{6}}\left\|v-\mathrm{P}_{N} v\right\|+\mathrm{N}^{-\frac{1}{3}}\left|v-\mathrm{P}_{N} v\right|_{1}\right)+\left\|\mathrm{P}_{N} v-v\right\|_{\mu} \\
& \leqslant c N^{\frac{1}{6}+\frac{\mu-\mathrm{r}}{2}}\|v\|_{\mathrm{r}, \mathrm{A}} .
\end{aligned}
$$

Consequently, the proof is complete. 
Now we give the two fully discrete schemes for Hermite pseudospectral method and modified Hermite method, respectively. Let $\tau$ be the step-size in variable $t, t_{k}=k \tau(k=0,1, \cdots, M ; M=[T / \tau])$, $u^{k}=u\left(x, t_{k}\right), u^{k+\frac{1}{2}}=u\left(x, t_{k+\frac{1}{2}}\right)$, and

$$
\overline{\partial_{t}} u^{k}=\frac{u^{k+1}-u^{k}}{\tau}, \quad u^{\hat{k}}=\frac{u^{k+1}+u^{k}}{2} .
$$

The fully discrete Hermite pseudospectral scheme for equations (1.1)-(1.4) is to find $s_{N}^{k}(x, t), l_{N}^{k}(x, t) \in \mathcal{H}_{N}$ such that for any $v \in \mathcal{H}_{N}$, we have

$$
\left\{\begin{array}{l}
i\left(\partial_{t} s_{N}^{k}, v\right)-\left(s_{N x}^{\hat{k}}, v_{x}\right)_{N}=\alpha\left(s_{N}^{\hat{k}} l_{N}^{k}, v\right)_{N}+\left(f^{k+\frac{1}{2}}, v\right)_{N}, \quad k=0,1, \cdots, M-1, \\
\left(\partial_{t} l_{N}^{k}, v\right)+\beta\left(\mid s_{N}^{k} l_{x}^{2}, v\right)_{N}=\left(g^{k+\frac{1}{2}}, v\right)_{N}, \quad k=0,1, \ldots, M-1, \\
s_{N}^{0}=I_{N} s_{0}, \quad l_{N}^{0}=I_{N} l_{0} .
\end{array}\right.
$$

Set

$$
u_{\hat{\mathrm{t}}}^{k}=\frac{u^{k+1}-u^{k-1}}{\tau}, \quad u^{\bar{k}}=\frac{u^{k+1}+u^{k-1}}{2} .
$$

The fully discrete modified Hermite spectral scheme for equations (1.1)-(1.4) is to find $s_{N}^{k}(x, t)$, $l_{\mathrm{N}}^{k}(x, t) \in \mathcal{H}_{N}$ such that for any $v \in \mathcal{H}_{N}$, we have

$$
\left\{\begin{array}{l}
i\left(s_{N \hat{t}}^{k}, v\right)-\left(s_{N x}^{\bar{k}}, v_{x}\right)=\alpha\left(s_{N}^{\bar{k}} l_{N}^{k}, v\right)_{N}+\left(f^{k}, v\right)_{N}, \quad k=1,2, \cdots, M-1, \\
\left(l_{N \hat{t}}^{k}, v\right)+\beta\left(\left(\left|s_{N}^{k}\right|^{2}\right)_{x}, v\right)_{N}=\left(g^{k}, v\right)_{N}, \quad k=1,2, \cdots, M-1, \\
s_{N}^{0}=I_{N} s_{0}, \quad l_{N}^{0}=I_{N} l_{0}, \\
s_{N}^{1}=I_{N}\left(s_{0}+i \tau\left(s_{0 x x}-\alpha s_{0} l_{0}-f^{0}\right)\right), l_{N}^{1}=I_{N}\left(l_{0}-\tau\left(\beta\left|s_{0}\right|_{x}^{2}-g^{0}\right)\right) .
\end{array}\right.
$$

\section{Hermite pseudospectral method for LS equations}

In this section, we just prove the convergence of the scheme (2.7)-(2.9). In order to obtain the error estimates of approximate solutions, we first introduce the following two lemmas:

Lemma 3.1. If $u, v \in H_{A}^{r}(\mathbf{R})$ and integer $r \geqslant 1$, then there exists a constant $C_{r}=2^{r}$ such that

$$
\|u v\|_{r, A} \leqslant C_{r}\|u\|_{r, A}\|v\|_{r, A} .
$$

Proof. We proceed by induction. For $r=1$, using the definition of $\|u\|_{r, A}$ and the relation $\|u\|_{\infty} \leqslant\|u\|_{1} \leqslant$ $\|u\|_{r, A}(r \geqslant 1)$, we have

$$
\begin{aligned}
\|u v\|_{1, A}^{2} & =\left\|\left(x^{2}+1\right)^{\frac{1}{2}}(u v)\right\|^{2}+\left\|(u v)_{x}\right\|^{2} \\
& \leqslant\|u\|_{\infty}^{2}\left\|\left(x^{2}+1\right)^{\frac{1}{2}} v\right\|^{2}+2\left(\|u\|_{\infty}^{2}\left\|v_{x}\right\|^{2}+\|v\|_{\infty}^{2}\left\|u_{x}\right\|^{2}\right) \\
& \leqslant 2\|u\|_{\infty}^{2}\|v\|_{1, A}^{2}+2\|v\|_{\infty}^{2}\left\|u_{x}\right\|^{2} \\
& \leqslant 4\|u\|_{1, A}^{2}\|v\|_{1, A}^{2} .
\end{aligned}
$$

Now we suppose $\|u v\|_{m, A}^{2} \leqslant 4^{m}\|u\|_{m, A}^{2}\|v\|_{m, A}^{2}$ holds for $r=m$. For $r=m+1$, we obtain

$$
\begin{aligned}
\|u v\|_{m+1, A}^{2} & =\left\|\left(x^{2}+1\right)^{\frac{m+1}{2}}(u v)\right\|^{2}+\left\|(u v)_{x}\right\|_{m, A}^{2} \\
& \leqslant\|u\|_{\infty}^{2}\left\|\left(x^{2}+1\right)^{\frac{m+1}{2}} v\right\|^{2}+2 \cdot 4^{m}\left(\|u\|_{m, A}^{2}\left\|v_{x}\right\|_{m, A}^{2}+\|v\|_{m, A}^{2}\left\|u_{x}\right\|_{m, A}^{2}\right) \\
& \leqslant 2 \cdot 4^{m}\left(\|u\|_{m, A}^{2}\|v\|_{m+1, A}^{2}+\|v\|_{m, A}^{2}\left\|u_{x}\right\|_{m, A}^{2}\right) \\
& \leqslant 4^{m+1}\|u\|_{m+1, A}^{2}\|v\|_{m+1, A}^{2} .
\end{aligned}
$$

Finally, we accomplish the proof. 
Lemma 3.2 ([13]). Assume that

1. $E^{k}, \rho^{k}(k=0,1, \cdots, M)$ are nonnegative grid functions, $\rho^{k}$ is increasing, and $\epsilon, C$ are positive constants;

2. for any $1 \leqslant n \leqslant M$, if $\max _{0 \leqslant k \leqslant n-1} E^{k} \leqslant \epsilon$, then $E^{n} \leqslant \rho^{n}+C \tau \sum_{k=0}^{n-1} E^{k}$;

3. $E^{0} \leqslant \rho^{0}$ and $\rho^{\mathrm{M}} e^{\mathrm{CT}} \leqslant \epsilon$.

Then for any $0<\mathrm{n} \leqslant M, \mathrm{E}^{n} \leqslant \rho^{n \tau} e^{\mathrm{Cn} \tau}$.

Now we give the main result of this section.

Theorem 3.3. Assume that $\mathrm{N}$ is sufficiently big, $\tau$ is sufficiently small such that $\tau^{2} \mathrm{~N}^{\frac{1}{2}}$ is sufficiently small, $s \in \mathrm{L}^{\infty}\left(0, \mathrm{~T} ; \mathrm{H}_{\mathrm{A}}^{\mathrm{r}}(\mathbf{R})\right), \mathrm{l} \in \mathrm{L}^{\infty}\left(0, \mathrm{~T} ; \mathrm{H}_{\mathrm{A}}^{\mathrm{r}-2}(\mathbf{R})\right), \mathrm{l}_{\mathrm{t}} \in \mathrm{L}^{\infty}\left(0, \mathrm{~T} ; \mathrm{H}^{1}(\mathbf{R})\right) \cap \mathrm{L}^{2}\left(0, \mathrm{~T} ; \mathrm{H}_{\mathrm{A}}^{\mathrm{r}-2}(\mathbf{R})\right), \mathrm{s}_{\mathrm{t}} \in \mathrm{L}^{\infty}\left(0, \mathrm{~T} ; \mathrm{H}^{2}(\mathbf{R})\right) \cap$ $\mathrm{L}^{2}\left(0, \mathrm{~T} ; \mathrm{H}_{A}^{\mathrm{r}}(\mathbf{R})\right), \mathrm{f}, \mathrm{g} \in \mathrm{L}^{\infty}\left(0, \mathrm{~T} ; \mathrm{H}_{A}^{\mathrm{r}-2}(\mathbf{R})\right), \mathrm{f}_{\mathrm{t}} \in \mathrm{L}^{2}\left(0, \mathrm{~T} ; \mathrm{H}_{\mathrm{A}}^{\mathrm{r}-2}(\mathbf{R})\right)$ for $\mathrm{r} \geqslant 3$ and $\mathrm{l}_{\mathrm{tt}}, \mathrm{s}_{\mathrm{ttt}} \in \mathrm{L}^{2}\left(0, \mathrm{~T} ; \mathrm{L}^{2}(\mathbf{R})\right)$, $s_{\mathrm{tt}} \in \mathrm{L}^{\infty}\left(0, \mathrm{~T} ; \mathrm{L}^{2}(\mathbf{R})\right)$. Then we have

$$
\left\|s^{n}-s_{N}^{n}\right\|_{1, N}+\left\|l^{n}-l_{N}^{n}\right\| \leqslant C\left(\tau+N^{\frac{7}{6}-\frac{r}{2}}\right), \quad n=0,1,2, \cdots, M,
$$

where $\mathrm{C}$ is independent of $\mathrm{N}$ and $\tau$.

Proof. Let

$$
\left\{\begin{array}{l}
e^{k}=s_{N}^{k}-s^{k}=\left(s_{N}^{k}-P_{N} s^{k}\right)+\left(P_{N} s^{k}-s^{k}\right) \triangleq e_{1}^{k}+e_{2}^{k}, \\
\eta^{k}=l_{N}^{k}-l^{k}=\left(l_{N}^{k}-P_{N} l^{k}\right)+\left(P_{N} l^{k}-l^{k}\right) \triangleq \eta_{1}^{k}+\eta_{2}^{k} .
\end{array}\right.
$$

Using equations (1.1)-(1.4), (2.7)-(2.9), (2.4), and the definition of $P_{N}$, we obtain for any $v \in \mathcal{H}_{N}, k=$ $0,1, \cdots, M-1$

$$
\left\{\begin{aligned}
& i\left(\partial_{t} e_{1}^{k}, v\right)-\left(e_{1 x}^{\hat{k}}, v_{x}\right)_{N}= \alpha\left(I_{N}\left(s_{N}^{\hat{k}} l_{N}^{k}\right)-s^{k+\frac{1}{2}} l^{k+\frac{1}{2}}, v\right)+i\left(s_{t}^{k+\frac{1}{2}}-\partial_{t}^{-} s^{k}, v\right) \\
&+\left(\left(P_{N} s^{\hat{k}}\right)_{x}, v_{x}\right)_{N}-\left(s_{x}^{k+\frac{1}{2}}, v_{x}\right)+\left(I_{N} f^{k+\frac{1}{2}}-f^{k+\frac{1}{2}}, v\right), \\
&\left(\partial_{t} \eta_{1}^{k}, v\right)+\beta\left(I_{N}\left(\left|s_{N}^{k}\right|_{x}^{2}\right)-\left|s^{k+\frac{1}{2}}\right|_{x}^{2}, v\right)=\left(l_{t}^{k+\frac{1}{2}}-\partial_{t} l^{k}, v\right)+\left(I_{N} g^{k+\frac{1}{2}}-g^{k+\frac{1}{2}}, v\right), \\
& e_{1}^{0}=\left(I_{N}-P_{N}\right) s_{0}, \quad \eta_{1}^{0}=\left(I_{N}-P_{N}\right) l_{0} .
\end{aligned}\right.
$$

For given integer $n, 1 \leqslant n \leqslant M$, we assume that

$$
\max _{0 \leqslant k \leqslant n-1}\left\|e_{1}^{k}\right\|_{1, N}^{2}+\left\|\eta_{1}^{k}\right\|^{2} \leqslant N^{-\frac{1}{2}}
$$

Then using Lemma 2.1 and equation (3.4), it gives

$$
\left\|e_{1}^{k}\right\|_{\infty} \leqslant\left\|e_{1}^{k}\right\|^{\frac{1}{2}}\left\|e_{1 x}^{k}\right\|^{\frac{1}{2}} \leqslant a_{0} N^{\frac{1}{4}}\left\|e_{1}^{k}\right\| \leqslant a_{0}, \quad k=0,1, \cdots, n-1 .
$$

Similarly, we have

$$
\left\|\eta_{1}^{k}\right\|_{\infty} \leqslant a_{0}, \quad k=0,1, \cdots, n-1 .
$$

In view of equations (3.5), (3.6), and Lemma 2.2, we obtain for $0 \leqslant k \leqslant n-1$

$$
\begin{aligned}
& \left\|s_{N}^{k}\right\|_{\infty} \leqslant\left\|e_{1}^{k}\right\|_{\infty}+\left\|P_{N} s^{k}\right\|_{\infty} \leqslant a_{0}+\left\|P_{N} s^{k}\right\|^{\frac{1}{2}}\left|P_{N} s^{k}\right|_{1}^{\frac{1}{2}} \leqslant a_{0}+c\|s\|_{L^{\infty}\left(0, T ; H_{A}^{1}(\mathbf{R})\right)} \triangleq a_{1}, \\
& \left\|l_{N}^{k}\right\|_{\infty} \leqslant a_{0}+c\|l\|_{L^{\infty}\left(0, T ; H_{A}^{1}(\mathbf{R})\right)} \triangleq a_{2} .
\end{aligned}
$$

In what follows, we estimate $\left\|e_{1}^{\mathfrak{n}}\right\|_{1, N}$ and $\left\|\eta_{1}^{\mathfrak{n}}\right\|$. Firstly, letting $v=e_{1}^{\hat{k}}$ in equation (3.1) and taking the imaginary part, we obtain

$$
\begin{aligned}
\frac{1}{2} \bar{\partial}_{t}\left\|e_{1}^{k}\right\|^{2}= & \alpha \operatorname{Im}\left(\operatorname{I}_{N}\left(s_{N}^{\hat{k}} l_{N}^{k}\right)-s^{k+\frac{1}{2}} l^{k+\frac{1}{2}}, e_{1}^{\hat{k}}\right)+\operatorname{Re}\left(s_{t}^{k+\frac{1}{2}}-\partial_{t}^{-} s^{k}, e_{1}^{\hat{k}}\right) \\
& \left.+\operatorname{Im}\left(\left(P_{N} s^{\hat{k}}\right)_{x}, e_{1 x}^{\hat{k}}\right)_{N}-\left(s_{x}^{k+\frac{1}{2}}, e_{1 x}^{\hat{k}}\right)\right)+\operatorname{Im}\left(I_{N} f^{k+\frac{1}{2}}-f^{k+\frac{1}{2}}, v\right) .
\end{aligned}
$$


According to Hölder inequality and equation (3.7), one has

$$
\begin{aligned}
& \alpha \operatorname{Im}\left(I_{N}\left(s_{N}^{\hat{k}} l_{N}^{k}\right)-s^{k+\frac{1}{2}} l^{k+\frac{1}{2}}, e_{1}^{\hat{k}}\right) \\
& =\alpha \operatorname{Im}\left(I_{N}\left[l_{N}^{k} e_{2}^{\hat{k}}+s^{\hat{k}}\left(\eta_{1}^{k}+\eta_{2}^{k}\right)\right]+\left(I_{N}-I\right) s^{\hat{k}} l^{k}+s^{\hat{k}}\left(l^{k}-l^{k+\frac{1}{2}}\right)+l^{k+\frac{1}{2}}\left(s^{\hat{k}}-s^{k+\frac{1}{2}}\right), e_{1}^{\hat{k}}\right) \\
& \leqslant a_{3}\left(\left\|e_{2}^{\hat{k}}\right\|_{N}+\left\|\eta_{1}^{k}\right\|+\left\|\eta_{2}^{k}\right\|_{N}+\left\|\left(I_{N}-I\right) s^{\hat{k}} l^{k}\right\|+\left\|l^{k}-l^{k+\frac{1}{2}}\right\|+\left\|s^{\hat{k}}-s^{k+\frac{1}{2}}\right\|\right)\left\|e_{1}^{\hat{k}}\right\|,
\end{aligned}
$$

$\operatorname{Re}\left(s_{t}^{k+\frac{1}{2}}-\overline{\partial_{t}} s^{k}, e_{1}^{\hat{k}}\right) \leqslant\left\|s_{t}^{k+\frac{1}{2}}-\overline{\partial t}_{t} s^{k}\right\|\left\|e_{1}^{\hat{k}}\right\|$,

and

$$
\operatorname{Im}\left(I_{N} f^{k+\frac{1}{2}}-f^{k+\frac{1}{2}}, e_{1}^{\hat{k}}\right) \leqslant\left\|I_{N} f^{k+\frac{1}{2}}-f^{k+\frac{1}{2}}\right\|\left\|e_{1}^{\hat{k}}\right\|,
$$

where $a_{3}=\max \left\{\alpha\|s\|_{L^{\infty}\left(0, T ; H^{1}(\mathbf{R})\right)}, \sqrt{2} \alpha\|l\|_{L^{\infty}\left(0, T ; H^{1}(\mathbf{R})\right)}, \alpha a_{2}, \alpha, \sqrt{2}\right\}$. For the third term on the right hand of equation (3.8), using equation (2.4), the property $\left\|u_{N x}\right\|_{N} \leqslant\left\|u_{N x}\right\|$ due to $I_{N} u_{N+1}=P_{N} u_{N+1}$, the definition of $P_{N}$, Hölder inequality, and Lemma 2.1, we infer that

$$
\begin{aligned}
\operatorname{Im}\left(\left(\left(P_{N} s^{\hat{k}}\right)_{x}, e_{1 x}^{\hat{k}}\right)_{N}-\left(s_{x}^{k+\frac{1}{2}}, e_{1 x}^{\hat{k}}\right)\right) & =\operatorname{Im}\left(\left(I_{N}\left(P_{N} s^{\hat{k}}\right)_{x}-P_{N} s_{x}^{\hat{k}}\right)+\left(P_{N}-I\right) s_{x}^{\hat{k}}+\left(s_{x}^{\hat{k}}-s_{x}^{k+\frac{1}{2}}\right), e_{1 x}^{\hat{k}}\right) \\
& =\operatorname{Im}\left(P_{N}\left(\left(P_{N} s^{\hat{k}}\right)_{x}-s_{x}^{\hat{k}}\right)+\left(P_{N}-I\right) s_{x}^{\hat{k}}+\left(s_{x}^{\hat{k}}-s_{x}^{k+\frac{1}{2}}\right), e_{1 x}^{\hat{k}}\right) \\
& \leqslant\left(c N^{\frac{1}{2}}\left\|\left(\left(P_{N}-I\right)^{\hat{k}}\right)_{x}\right\|+\left\|\left(\left(P_{N}-I\right)_{x}^{\hat{k}}\right)_{x}\right\|+\left\|s_{x x}^{\hat{k}}-s_{x x}^{k+\frac{1}{2}}\right\|\right)\left\|e_{1}^{\hat{k}}\right\| .
\end{aligned}
$$

Substituting the above four estimates into equation (3.8), we deduce that

$$
\begin{aligned}
\overline{\partial_{t}}\left\|e_{1}^{k}\right\| \leqslant & a_{3}\left(\left\|s^{\hat{k}}-s^{k+\frac{1}{2}}\right\|_{2}+\left\|l^{k}-l^{k+\frac{1}{2}}\right\|+\left\|s_{t}^{k+\frac{1}{2}}-\overline{\partial_{t}} s^{k}\right\|+\left\|e_{2}^{\hat{k}}\right\|_{N}+\left\|\eta_{2}^{k}\right\|_{N}+\left\|\left(I_{N}-I\right)\left(s^{\hat{k}} l^{k}\right)\right\|\right. \\
& \left.+c N^{\frac{1}{2}}\left\|\left(\left(P_{N}-I\right) s^{\hat{k}}\right)_{x}\right\|+\left\|\left(\left(P_{N}-I\right) s_{\chi}^{\hat{k}}\right)_{x}\right\|+\left\|\left(I_{N}-I\right) f^{k+\frac{1}{2}}\right\|\right)+a_{3}\left\|\eta_{1}^{k}\right\| .
\end{aligned}
$$

By Taylor's expansion and Hölder inequality, we find

$$
\begin{aligned}
\left\|s^{\hat{k}}-s^{k+\frac{1}{2}}\right\| & =\frac{1}{2}\left\|\int_{t_{k+\frac{1}{2}}}^{t_{k+1}} s_{t} d t-\int_{t_{k}}^{t_{k+\frac{1}{2}}} s_{t} d t\right\| \\
& \leqslant \frac{1}{2}\left\|\left(\int_{t_{k+\frac{1}{2}}}^{t_{k+1}} 1 d t\right)^{\frac{1}{2}}\left(\int_{t_{k+\frac{1}{2}}}^{t_{k+1}} s_{t}^{2} d t\right)^{\frac{1}{2}}+\left(\int_{t_{k}}^{t_{k+\frac{1}{2}}} 1 d t\right)^{\frac{1}{2}}\left(\int_{t_{k}}^{t_{k+\frac{1}{2}}} s_{t}^{2} d t\right)^{\frac{1}{2}}\right\| \\
& \leqslant \frac{\tau^{\frac{1}{2}}}{2}\left(\int_{t_{k}}^{t_{k+1}}\left\|s_{t}\right\|^{2} d t\right)^{\frac{1}{2}} \leqslant \frac{\tau}{2} \max _{t}\left\|s_{t}\right\|, \\
\left\|s_{t}^{k+\frac{1}{2}}-\overline{\partial_{t}} s^{k}\right\|^{2} & =\frac{1}{\tau}\left\|\int_{t_{k+\frac{1}{2}}^{t_{k+1}}}^{t_{k+1}}\left(t_{k} t\right) s_{t t} d t+\int_{t_{k}}^{t_{k+\frac{1}{2}}}\left(t-t_{k}\right) s_{t t} d t\right\| \leqslant \frac{\tau}{2 \sqrt{3}} \max _{t}\left\|s_{t t}\right\|, \\
\left\|s_{x x}^{\hat{k}}-s_{x x}^{k+\frac{1}{2}}\right\| & \leqslant \frac{\tau}{2} \max _{t}\left\|s_{x x t}\right\| \text { and }\left\|l^{k}-l^{k+\frac{1}{2}}\right\|=\left\|\int_{t_{k}}^{t_{k+\frac{1}{2}}} l_{t} d t\right\| \leqslant \frac{\tau}{2} \max _{t}\left\|l_{t}\right\| .
\end{aligned}
$$

Using equation (2.6) and Lemma 2.2, we infer that

$$
\left\|\eta_{2}^{k}\right\|_{N} \leqslant c N^{\frac{1}{6}}\left\|\left(P_{N}-I\right) l^{k}\right\|+c N^{-\frac{1}{3}}\left\|\left(\left(P_{N}-I\right) l^{k}\right)_{x}\right\| \leqslant c N^{\frac{7}{6}-\frac{r}{2}} \max _{t}\|l\|_{r-2, A} .
$$

Similarly, we have

$$
\left\|e_{2}^{\hat{k}}\right\|_{N} \leqslant c N^{\frac{7}{6}-\frac{r}{2}} \max _{t}\|s\|_{r, A}
$$


According to Lemma 2.2, Theorem 2.3, and Lemma 3.1, it gives

$$
\begin{aligned}
N^{\frac{1}{2}}\left\|\left(\left(P_{N}-I\right) s^{\hat{k}}\right)_{x}\right\| \leqslant c N^{\frac{7}{6}-\frac{r}{2}} \max _{t}\|s\|_{r, A}, & \left\|\left(\left(P_{N}-I\right) s_{x}^{\hat{k}}\right)_{x}\right\| \leqslant c N^{\frac{7}{6}-\frac{r}{2}} \max _{t}\|s\|_{r, A}, \\
\left\|\left(I_{N}-I\right)\left(s^{\hat{k}} l^{k}\right)\right\| \leqslant c N^{\frac{7}{6}-\frac{r}{2}} \max _{t}\|s\|_{r-2, A} \max _{t}\|l\|_{r-2, A}, & \left\|\left(I_{N}-I\right) f^{k+\frac{1}{2}}\right\|^{2} \leqslant c N^{\frac{7}{6}-\frac{r}{2}} \max _{t}\|f\|_{r-2, A} .
\end{aligned}
$$

Substituting all the above estimates into equation (3.9), then evaluating the sum for $k$ from 0 to $n-1$ and using Hölder inequality, we deduce

$$
\left\|e_{1}^{n}\right\|^{2} \leqslant 4\left\|e_{1}^{0}\right\|^{2}+a_{3}^{2} a_{4} T \tau^{2}+c a_{3}^{2} a_{5} T^{2} N^{2-r}+4 a_{3}^{2} T \tau \sum_{k=0}^{n-1}\left\|\eta_{1}^{k}\right\|^{2},
$$

where $a_{4}=\max _{\mathfrak{t}}\left(\left\|s_{\mathrm{t}}\right\|_{2}^{2}+\left\|s_{\mathrm{tt}}\right\|^{2}+\left\|l_{\mathrm{t}}\right\|^{2}\right)$ and

$$
a_{5}=\max _{t}\left(\|s\|_{r, A}^{2}+\|l\|_{r-2, A}^{2}+\|f\|_{r-2, A}^{2}\right)+\max _{t}\|s\|_{r-2, A}^{2} \max _{t}\|l\|_{r-2, A}^{2} .
$$

To estimate $\left\|\eta_{1}^{k}\right\|$, letting $v=\eta_{1}^{\hat{k}}$ in equation (3.2), we find

$$
\begin{aligned}
\bar{\partial}_{t}\left\|\eta_{1}^{k}\right\| & \leqslant \beta\left\|I_{N}\left(\left|s_{N}^{k}\right|^{2}\right)_{x}-\left(\left|s^{k}\right|^{2}\right)_{x}\right\|+\left\|l_{t}^{k+\frac{1}{2}}-\bar{\partial}_{t} l^{k}\right\|+\left\|\left(I_{N}-I\right) g^{k+\frac{1}{2}}\right\| \\
& \leqslant 2 \beta\left\|I_{N}\left(s_{N}^{k}\left(\bar{s}_{N x}^{k}-\bar{s}_{x}^{k}\right)+\bar{s}_{x}^{k}\left(s_{N}^{k}-s^{k}\right)\right)\right\|+\beta\left\|\left(I_{N}-I\right)\left(\left|s^{k}\right|^{2}\right)_{x}\right\|+\left\|l_{t}^{k+\frac{1}{2}}-\bar{\partial}_{t} l^{k}\right\|+\left\|\left(I_{N}-I\right) g^{k+\frac{1}{2}}\right\| \\
& \leqslant a_{6}\left(\left\|e_{2}^{k}\right\|_{1, N}+\left\|\left(I_{N}-I\right)\left(s_{x}^{k} \bar{s}^{k}\right)\right\|+\left\|l_{t}^{k+\frac{1}{2}}-\overline{\partial_{t}} l^{k}\right\|+\left\|\left(I_{N}-I\right) g^{k+\frac{1}{2}}\right\|\right)+a_{6}\left\|e_{1}^{k}\right\|_{1, N},
\end{aligned}
$$

where $a_{6}=\max \left\{2 \sqrt{2} \beta a_{1}, 2 \sqrt{2} \beta\|s\|_{L^{\infty}\left(0, T ; H^{2}(\mathbf{R})\right)}, 2 \beta, 1\right\}$. Taking the sum for $\mathrm{k}$ from 0 to $n-1$, then using Hölder inequality, Taylor's expansion, Theorem 2.3, equation (2.6) and Lemmas 2.2, 3.1, we deduce

$$
\left\|\eta_{1}^{n}\right\|^{2} \leqslant 4\left\|\eta_{1}^{0}\right\|^{2}+a_{6}^{2} a_{7} T \tau^{2}+c a_{6}^{2} a_{8} T^{2} N^{2-r}+4 a_{6}^{2} T \tau \sum_{k=0}^{n-1}\left\|e_{1}^{k}\right\|_{1, N}^{2}
$$

where

$$
a_{7}=\int_{0}^{T}\left\|l_{t t}\right\|^{2} d t, \quad a_{8}=\max _{t}\left(\|s\|_{r, A}^{2}+\|s\|_{r-1, A}^{4}+\|g\|_{r-2, A}^{2}\right) .
$$

To estimate $\left\|e_{1 x}^{k}\right\|_{N}$, letting $v=\overline{\partial t}_{t} e_{1}^{k}$ in equation (3.1) and considering the real part, we infer that

$$
\begin{aligned}
\frac{1}{2} \overline{\partial_{t}}\left\|e_{1 x}^{k}\right\|_{N}^{2}= & -\alpha \operatorname{Re}\left(I_{N}\left(s_{N}^{\hat{k}} l_{N}^{k}\right)-s^{k+\frac{1}{2}} l^{k+\frac{1}{2}}, \overline{\partial_{t}} e_{1}^{k}\right)+\operatorname{Im}\left(s_{t}^{k+\frac{1}{2}}-\overline{\partial_{t}} s^{k}, \overline{\partial_{t}} e_{1}^{k}\right) \\
& +\operatorname{Re}\left(\left(s_{x}^{k+\frac{1}{2}}, \overline{\partial_{t}} e_{1 x}^{k}\right)-\left(\left(P_{N} s^{\hat{k}}\right)_{x}, \overline{\partial_{t}} e_{1 x}^{k}\right)_{N}\right)+\operatorname{Re}\left(I_{N} f^{k+\frac{1}{2}}-f^{k+\frac{1}{2}}, \overline{\partial_{t}} e_{1}^{k}\right) \\
\leqslant & a_{3}\left(\left\|e_{1}^{\hat{k}}\right\|+\left\|\eta_{1}^{k}\right\|+\left\|e_{2}^{\hat{k}}\right\|_{N}+\left\|\eta_{2}^{k}\right\|_{N}+\left\|s^{\hat{k}}-s^{k+\frac{1}{2}}\right\|_{2}+\left\|l^{k}-l^{k+\frac{1}{2}}\right\|+\left\|s_{t}^{k+\frac{1}{2}}-\overline{\partial_{t}} s^{k}\right\|\right. \\
& \left.+\left\|\left(\left(P_{N}-I\right) s_{x}^{\hat{k}}\right)_{x}\right\|+c N^{\frac{1}{2}}\left\|\left(\left(P_{N}-I\right) s^{\hat{k}}\right)_{x}\right\|+\left\|\left(I_{N}-I\right)\left(s^{\hat{k}^{k}}\right)\right\|+\left\|\left(I_{N}-I\right) f^{k+\frac{1}{2}}\right\|\right)\left\|\partial_{t} e_{1}^{k}\right\| .
\end{aligned}
$$

Taking the sum for $\mathrm{k}$ from 0 to $\mathrm{n}-1$, using Hölder inequality, Taylor's expansion, Theorem 2.3, equation (2.6) and Lemmas 2.2, 3.1, we obtain

$$
\left\|e_{1 x}^{n}\right\|_{N}^{2} \leqslant\left\|e_{1 x}^{0}\right\|_{N}^{2}+a_{3} a_{4} \tau^{2}+c a_{3} a_{5} N^{2-r}+a_{9} \tau \sum_{k=0}^{n-1}\left(\left\|e_{1}^{k}\right\|^{2}+\left\|\eta_{1}^{k}\right\|^{2}+\left\|\partial_{t} e_{1}^{k}\right\|^{2}\right)+\frac{1}{2}\left\|e_{1}^{n}\right\|^{2},
$$

where $a_{9}=\frac{a_{3}^{2}}{4}+\frac{\sqrt{10} a_{3}}{4}$. To estimate $\left\|\partial_{t} e_{1}^{k}\right\|$, setting $w^{k}=\partial_{t} e_{1}^{k}$, making forward difference quotient for 
equation (3.1), then taking $v=w^{\hat{k}}$ and looking at the imaginary part, it gives

$$
\begin{aligned}
\frac{1}{2} \partial_{t}\left\|w^{k}\right\|^{2}= & \frac{\alpha}{\tau} \operatorname{Im}\left(\operatorname{I}_{N}\left(s_{N}^{\widehat{k+1}} l_{N}^{k+1}-s_{N}^{\hat{k}} l_{N}^{k}\right)-\left(s^{k+\frac{3}{2}} l^{k+\frac{3}{2}}-s^{k+\frac{1}{2}} l^{k+\frac{1}{2}}\right), w^{\hat{k}}\right) \\
& +\operatorname{Re}\left(\overline{\partial_{t}} s_{t}^{k+\frac{1}{2}}-\overline{\partial_{t}} \overline{\partial_{t}} s^{k}, w^{\hat{k}}\right)+\operatorname{Im}\left(\left(\left(P_{N} \overline{\partial_{t}} s^{\hat{k}}\right)_{x}, w_{x}^{\hat{k}}\right)_{N}-\left(\partial_{t} s_{x}^{k+\frac{1}{2}}, w_{x}^{\hat{k}}\right)\right) \\
& +\operatorname{Im}\left(\left(I_{N}-I\right) \partial_{t} f^{k+\frac{1}{2}}, w^{\hat{k}}\right) .
\end{aligned}
$$

Now we estimate the first term and the third term on the right hand of equation (3.13), respectively. In view of Hölder inequality, we have

$$
\begin{aligned}
& \alpha \frac{1}{\tau} \operatorname{Im}\left(\mathrm{I}_{\mathrm{N}}\left(\mathrm{s}_{\mathrm{N}}^{\widehat{\mathrm{k}+1}} l_{\mathrm{N}}^{\mathrm{k}+1}-s_{\mathrm{N}}^{\hat{k}} l_{\mathrm{N}}^{\mathrm{k}}\right)-\left(\mathrm{s}^{\mathrm{k}+\frac{3}{2}} l^{k+\frac{3}{2}}-s^{k+\frac{1}{2}} l^{k+\frac{1}{2}}\right), w^{\hat{k}}\right) \\
& =\alpha \operatorname{Im}\left(\operatorname{I}_{N}\left(l_{N}^{k+1} \bar{\partial}_{t} s_{N}^{\hat{k}}+s_{N}^{\hat{k}} \bar{\partial}_{t} l_{N}^{k}\right)-\left(l^{k+\frac{3}{2}} \overline{\partial_{t}} s^{k+\frac{1}{2}}+s^{k+\frac{1}{2}} \bar{\partial}_{t} l^{k+\frac{1}{2}}\right), w^{\hat{k}}\right) \\
& =\alpha \operatorname{Im}\left(\operatorname{I}_{N}\left[\left(l_{N}^{k+1} \partial_{t} s_{N}^{\hat{k}}+s_{N}^{\hat{k}} \partial_{t} l_{N}^{k}\right)-\left(l^{k+1} \partial_{t} s^{\hat{k}}+s^{\hat{k}} \overline{\partial_{t}} l^{k}\right)\right]+\left(I_{N}-I\right)\left(l^{k+1} \partial_{t} s^{\hat{k}}+s^{\hat{k}} \partial_{t} l^{k}\right)\right. \\
& \left.+l^{k+1}\left(\overline{\partial_{t}} s^{\hat{k}}-\overline{\partial_{t}} s^{k+\frac{1}{2}}\right)+\overline{\partial_{t}} s^{k+\frac{1}{2}}\left(l^{k+1}-l^{k+\frac{3}{2}}\right)+\overline{\partial_{t}} l^{k}\left(s^{\hat{k}}-s^{k+\frac{1}{2}}\right)+s^{k+\frac{1}{2}}\left(\partial_{t} l^{k}-\partial_{t} l^{k+\frac{1}{2}}\right), w^{\hat{k}}\right) \\
& =\alpha \operatorname{Im}\left(\operatorname{I}_{N}\left[l_{N}^{k+1} \bar{\partial}_{t} e_{2}^{\hat{k}}+\bar{\partial}_{t} s^{\hat{k}}\left(\eta_{1}^{k+1}+\eta_{2}^{k+1}\right)+s_{N}^{\hat{k}}\left(\bar{\partial}_{t} \eta_{1}^{k}+\bar{\partial}_{t} \eta_{2}^{k}\right)+\bar{\partial}_{t} l^{k}\left(e_{1}^{\hat{k}}+e_{2}^{\hat{k}}\right)\right], w^{\hat{k}}\right) \\
& +\alpha \operatorname{Im}\left(\left(I_{N}-I\right)\left(l^{k+1} \overline{\partial_{t}} s^{\hat{k}}+s^{\hat{k}} \bar{\partial}_{t} l^{k}\right)+l^{k+1}\left(\overline{\partial_{t}} s^{\hat{k}}-\partial_{t} s^{k+\frac{1}{2}}\right)+\partial_{t} s^{k+\frac{1}{2}}\left(l^{k+1}-l^{k+\frac{3}{2}}\right), w^{\hat{k}}\right) \\
& +\left(\partial_{t} l^{k}\left(s^{\hat{k}}-s^{k+\frac{1}{2}}\right)+s^{k+\frac{1}{2}}\left(\partial_{t} l^{k}-\partial_{t} l^{k+\frac{1}{2}}\right), w^{\hat{k}}\right) \\
& \leqslant a_{10}\left(\left\|e_{1}^{\hat{k}}\right\|+\left\|\eta_{1}^{k+1}\right\|+\left\|\bar{\partial}_{t} \eta_{1}^{k}\right\|+\left\|e_{2}^{\hat{k}}\right\|_{N}+\left\|\eta_{2}^{k+1}\right\|_{N}+\left\|\bar{\partial}_{t} e_{2}^{\hat{k}_{1}}\right\|_{N}+\left\|\bar{\partial}_{t} \eta_{2}^{k}\right\|_{N}+\left\|\left(I_{N}-I\right)\left(l^{k+1} \bar{\partial}_{t} s^{\hat{k}}\right)\right\|\right. \\
& \left.+\left\|\left(I_{N}-I\right)\left(s^{\hat{k}} \overline{\partial_{t}} l^{k}\right)\right\|+\left\|\overline{\partial_{t}} s^{\hat{k}}-\overline{\partial_{t}} s^{k+\frac{1}{2}}\right\|+\left\|s^{\hat{k}}-s^{k+\frac{1}{2}}\right\|+\left\|l^{k+1}-l^{k+\frac{3}{2}}\right\|+\left\|\partial_{t} l^{k}-\partial_{t} l^{k+\frac{1}{2}}\right\|\right)\left\|w^{\hat{k}}\right\|,
\end{aligned}
$$

where

$$
a_{10}=\max \left\{\alpha a_{1}, a_{3}, \alpha\left\|l_{t}\right\|_{L^{\infty}\left(0, T ; L^{2}(\mathbf{R})\right)}^{\frac{1}{2}}\left\|l_{x t}\right\|_{L^{\infty}\left(0, T ; L^{2}(\mathbf{R})\right)}^{\frac{1}{2}}, \alpha\left\|s_{t}\right\|_{L^{\infty}\left(0, T ; L^{2}(\mathbf{R})\right)}^{\frac{1}{2}}\left\|s_{x t}\right\|_{L^{\infty}\left(0, T ; L^{2}(\mathbf{R})\right\}}^{\frac{1}{2}}\right\} .
$$

Similarly, we have

$$
\begin{gathered}
\operatorname{Re}\left(\overline{\partial_{t}} s_{t}^{k+\frac{1}{2}}-\overline{\partial_{t}} \overline{\partial_{t}} s^{k}, w^{\hat{k}}\right) \leqslant\left\|\bar{\partial}_{t} s_{t}^{k+\frac{1}{2}}-\overline{\partial_{t}}{\overline{\partial_{t}}} s^{k}\right\|\left\|w^{\hat{k}}\right\|, \\
\operatorname{Im}\left(\left(I_{N}-I\right) \bar{\partial}_{t} f^{k+\frac{1}{2}}, w^{\hat{k}}\right) \leqslant\left\|\left(I_{N}-I\right) \partial_{t} f^{k+\frac{1}{2}}\right\|\left\|w^{\hat{k}}\right\|, \\
\operatorname{Im}\left(\left(\left(P_{N} \overline{\partial_{t}} s^{\hat{k}}\right)_{x}, w_{x}^{\hat{k}}\right)_{N}-\left(\overline{\partial_{t}} s_{x}^{k+\frac{1}{2}}, w_{x}^{\hat{k}}\right)\right) \\
=\operatorname{Im}\left(I_{N}\left(P_{N} \overline{\partial_{t}} s^{\hat{k}}\right)_{x}-P_{N}\left(\overline{\partial_{t}} s_{x}^{\hat{k}}\right)+\left(P_{N}-I\right) \partial_{t} s_{x}^{\hat{k}}+\left(\bar{\partial}_{t} s_{x}^{k+\frac{1}{2}}-\bar{\partial}_{t} s_{x}^{k+\frac{1}{2}}\right), w_{x}^{\hat{k}}\right) \\
\leqslant\left(c N^{\frac{1}{2}}\left\|\left(\left(P_{N}-I\right) \bar{\partial}_{t} s^{\hat{k}}\right)_{x}\right\|+\left\|\left(\left(P_{N}-I\right) \partial_{t} s_{x}^{\hat{k}}\right)_{x}\right\|+\left\|\bar{\partial}_{t} s_{x x}^{\hat{k}}-\bar{\partial}_{t} s_{x x}^{k+\frac{1}{2}}\right\|\right)\left\|w^{\hat{k}}\right\| .
\end{gathered}
$$

Substituting the above estimates into equation (3.13) and using the estimates of $\left\|\partial_{t} \eta_{1}^{k}\right\|$

$$
\left.\left\|\bar{\partial}_{t} \eta_{1}^{k}\right\| \leqslant a_{6}\left(\left\|e_{1}^{k}\right\|_{1, N}+\left\|e_{2}^{k}\right\|_{1, N}+\left\|l_{t}^{k+\frac{1}{2}}-\bar{\partial}_{t} l^{k}\right\|+\left\|\left(I_{N}-I\right)\left(s_{x}^{k} \bar{s}^{k}\right)\right\|+\left\|\left(I_{N}-I\right) g^{k+\frac{1}{2}}\right\|\right)\right),
$$

we obtain

$$
\begin{aligned}
\bar{\partial}_{t}\left\|w^{k}\right\| \leqslant & a_{10}\left(a_{6}\left\|e_{1}^{k}\right\|_{1, N}+\left\|e_{1}^{\hat{k}}\right\|+\left\|\eta_{1}^{k+1}\right\|+a_{6}\left\|e_{2}^{k}\right\|_{1, N}+\left\|e_{2}^{\hat{k}}\right\|_{N}+\left\|\eta_{2}^{k+1}\right\|_{N}+\left\|\overline{\partial_{t}} e_{2}^{\hat{k}_{k}}\right\|_{N}+\left\|\overline{\partial_{t}} \eta_{2}^{k}\right\|_{N}\right. \\
& +\left\|s^{\hat{k}}-s^{k+\frac{1}{2}}\right\|+\left\|l^{k+1}-l^{k+\frac{3}{2}}\right\|+\left\|\overline{\partial_{t}} l^{k}-\overline{\partial_{t}} l^{k+\frac{1}{2}}\right\|+a_{6}\left\|l_{t}^{k+\frac{1}{2}}-\overline{\partial_{t}} l^{k}\right\|+\left\|\overline{\partial_{t}} s^{\hat{k}}-\overline{\partial_{t}} s^{k+\frac{1}{2}}\right\|_{2} \\
& +\left\|\overline{\partial_{t}} s_{t}^{k+\frac{1}{2}}-\overline{\partial_{t}} \overline{\partial_{t}} s^{k}\right\|+\left\|\left(I_{N}-I\right)\left(l^{k+1} \overline{\partial_{t}} s^{\hat{k}}\right)\right\|+\left\|\left(I_{N}-I\right)\left(s^{\hat{k}} \overline{\partial_{t}} l^{k}\right)\right\|+a_{6}\left\|\left(I_{N}-I\right)\left(s_{x}^{k} \bar{s}^{k}\right)\right\| \\
& \left.+a_{6}\left\|\left(I_{N}-I\right) g^{k+\frac{1}{2}}\right\|+\left\|\left(I_{N}-I\right) \overline{\partial_{t}} f^{k+\frac{1}{2}}\right\|+c N^{\frac{1}{2}}\left\|\left(\left(P_{N}-I\right) \bar{\partial}_{t} s^{\hat{k}}\right)_{x}\right\|+\left\|\left(\left(P_{N}-I\right) \overline{\partial_{t}} \overline{s_{x}^{k}}\right)_{x}\right\|\right) .
\end{aligned}
$$


Taking the sum for $\mathrm{k}$ from 0 to $\mathrm{n}-2$, using Hölder inequality, Taylor's expansion, Theorem 2.3, equation (2.6), Lemmas 2.2, 3.1 and the estimates below

$$
\begin{aligned}
\left\|\partial_{t} l^{k}-\overline{\partial_{t}} l^{k+\frac{1}{2}}\right\|= & \frac{1}{\tau}\left\|\int_{t_{k+\frac{1}{2}}}^{t_{k+1}}\left(t_{k+1}-t\right) l_{t t} d t+\int_{t_{k}}^{t_{k+\frac{1}{2}}}\left(t-t_{k}\right) l_{t t} d t-\int_{t_{k+\frac{1}{2}}}^{t_{k+\frac{3}{2}}}\left(t_{k+\frac{3}{2}}-t\right) l_{t t} d t\right\| \\
\leqslant & \frac{\sqrt{5 \tau}}{2 \sqrt{3}}\left(\int_{t_{k}}^{t_{k+1}}\left\|l_{t t}\right\|^{2} d t+\int_{t_{k+\frac{1}{2}}}^{t_{k+\frac{3}{2}}}\left\|l_{t t}\right\|^{2} d t\right)^{\frac{1}{2}}, \\
\left\|\overline{\partial_{t}} s^{\hat{k}}-\overline{\partial_{t}} s^{k+\frac{1}{2}}\right\|= & \| \frac{1}{\tau}\left(\int_{t_{k+1}}^{t_{k+\frac{3}{2}}}\left(t_{k+\frac{3}{2}}-t\right) s_{t t t} d t-\int_{t_{k+1}}^{t_{k+\frac{1}{2}}}\left(t_{k+\frac{1}{2}}-t\right) s_{t t t} d t\right) \\
& -\frac{1}{2 \tau^{2}}\left(\int_{t_{k+1}}^{t_{k+2}}\left(t_{k+2}-t\right)^{2} s_{t t t} d t+\int_{t_{k+1}}^{t_{k}}\left(t_{k}-t\right)^{2} s_{t t t} d t\right) \| \\
\leqslant & \left(\frac{11 \tau}{60}\right)^{\frac{1}{2}}\left(\int_{t_{k+\frac{1}{2}}}^{t_{k+\frac{3}{2}}}\left\|s_{t t t}\right\|^{2} d t+\int_{t_{k}}^{t_{k+2}}\left\|s_{t t t}\right\|^{2} d t\right)^{\frac{1}{2}}, \\
\left\|\left(I_{N}-I\right)\left(s^{\hat{k}} \bar{\partial}_{t} l^{k+1}\right)\right\| \leqslant & c N^{\frac{7}{6}-\frac{r}{2}}\left\|s^{\hat{k}} \partial_{t} l^{k+1}\right\|_{r-2, A} \leqslant c N^{\frac{7}{6}-\frac{r}{2}} \max _{t}\|s\|_{r-2, A}\left(\frac{1}{\tau} \int_{t_{k+2}}^{t_{k+1}}\left\|l_{t}\right\|_{r-2, A}^{2}\right)^{\frac{1}{2}},
\end{aligned}
$$

and similarly,

$$
\begin{gathered}
\left\|\left(I_{N}-I\right)\left(l^{k+1} \partial_{t} s^{\hat{k}}\right)\right\| \leqslant c N^{\frac{7}{6}-\frac{r}{2}} \max _{t}\|l\|_{r-2, A}\left(\frac{1}{\tau} \int_{t_{k+2}}^{t_{k+1}}\left\|s_{t}\right\|_{r-2, A}^{2} d t\right)^{\frac{1}{2}}, \\
\left\|\left(I_{N}-I\right) \partial_{t} f^{k+\frac{1}{2}}\right\| \leqslant c N^{\frac{7}{6}-\frac{r}{2}}\left(\frac{1}{\tau} \int_{t_{k}}^{t_{k+1}}\left\|f_{t}\right\|_{r-2, A}^{2} d t\right)^{\frac{1}{2}}, \\
c N^{\frac{1}{2}}\left\|\left(\left(P_{N}-I\right) \bar{\partial}_{t} s^{\hat{k}}\right)_{x}\right\|+\left\|\left(\left(P_{N}-I\right) \partial_{t} s_{x}^{\hat{k}}\right)_{x}\right\| \leqslant c N^{\frac{7}{6}-\frac{r}{2}}\left(\frac{1}{\tau} \int_{t_{k}}^{t_{k+1}}\left\|s_{t}\right\|_{r, A}^{2} d t\right)^{\frac{1}{2}},
\end{gathered}
$$

thus we deduce

$$
\left\|\bar{\partial}_{t} e_{1}^{n-1}\right\|^{2} \leqslant 5\left\|\bar{\partial}_{t} e_{1}^{0}\right\|^{2}+5 a_{6}^{2} a_{10}^{2} a_{11} T \tau^{2}+c T^{2} a_{6}^{2} a_{10}^{2} a_{12} N^{2-r}+5 a_{6}^{2} a_{10}^{2} T \tau \sum_{k=0}^{n-1}\left(\left\|e_{1}^{k}\right\|_{1, N}^{2}+\left\|\eta_{1}^{k}\right\|^{2}\right),
$$

where $a_{11}=a_{4}+a_{7}+\int_{0}^{T}\left\|s_{t t t}\right\|^{2} d t$ and

$$
\begin{aligned}
a_{12}= & a_{5}+a_{8}+\int_{0}^{T}\left(\left\|s_{t}\right\|_{r, A}^{2}+\left\|l_{t}\right\|_{r-2, A}^{2}+\left\|f_{t}\right\|_{r-2, A}^{2}\right) d t \\
& +\max _{t}\|l\|_{r-2, A}^{2} \int_{0}^{T}\left\|s_{t}\right\|_{r-2, A}^{2} d t+\max _{t}\|s\|_{r-2, A}^{2} \int_{0}^{T}\left\|l_{t}\right\|_{r-2, A}^{2} d t .
\end{aligned}
$$

Substituting the above inequality into equation (3.12), we deduce that

$$
\begin{aligned}
\left\|e_{1 x}^{n}\right\|_{N}^{2} \leqslant & 5 a_{9} T\left\|\bar{\partial}_{t} e_{1}^{0}\right\|^{2}+\left\|e_{1 x}^{0}\right\|_{N}^{2}+\left(a_{3} a_{4}+5 a_{6}^{2} a_{10}^{2} a_{11} T^{2}\right) \tau^{2}+c\left(a_{3} a_{5}+a_{6}^{2} a_{10}^{2} a_{12} T^{3}\right) N^{2-r} \\
& +\left(a_{9}+5 a_{6}^{2} a_{10}^{2} T^{2}\right) \tau \sum_{k=0}^{n-1}\left(\left\|e_{1}^{k}\right\|_{1, N}^{2}+\left\|\eta_{1}^{k}\right\|^{2}\right)+\frac{1}{2}\left\|e_{1}^{n}\right\|^{2} .
\end{aligned}
$$

Adding equations (3.10), (3.11), and the above inequality up together, we deduce that

$$
\begin{aligned}
& \left\|e_{1}^{n}\right\|_{1, N}^{2}+\left\|\eta_{1}^{n}\right\|^{2} \\
& \quad \leqslant 8\left(\left\|e_{1}^{0}\right\|_{1, N}^{2}+\left\|\eta_{1}^{0}\right\|^{2}\right)+10 a_{9} T\left\|\bar{\partial}_{t} e_{1}^{0}\right\|^{2}+a_{14}\left(\tau^{2}+N^{2-r}\right)+a_{13} \tau \sum_{k=0}^{n-1}\left(\left\|e_{1}^{k}\right\|_{1, N}^{2}+\left\|\eta_{1}^{k}\right\|^{2}\right),
\end{aligned}
$$


where $a_{13}=2\left(4 a_{3}^{2} T+4 a_{6}^{2} T+a_{9}+5 a_{6}^{2} a_{10}^{2} T^{2}\right)$ and

$$
a_{14}=2 \max \left\{4 a_{3}^{2} a_{4} T+a_{6}^{2} a_{7} T+a_{3} a_{4}+5 a_{6}^{2} a_{10}^{2} a_{11} T^{2}, c a_{3}^{2} a_{5} T^{2}+c a_{6}^{2} a_{8} T^{2}+c\left(a_{3} a_{5}+a_{6}^{2} a_{10}^{2} a_{12} T^{3}\right)\right\} .
$$

Now we estimate the initial values. Using equation (3.3), the definition of $P_{N}$, triangular inequality, Lemmas 2.2, 3.1, Theorem 2.3, and the property $\left\|u_{N x}\right\|_{N} \leqslant\left\|u_{N x}\right\|$ due to $I_{N} u_{N+1}=P_{N} u_{N+1}$, we obtain

$$
\left\|e_{1}^{0}\right\|_{1, N}^{2} \leqslant\left\|e_{1}^{0}\right\|_{1}^{2} \leqslant 2\left(\left\|\left(I_{N}-I\right) s_{0}\right\|_{1}^{2}+\left\|\left(P_{N}-I\right) s_{0}\right\|_{1}^{2}\right) \leqslant \mathrm{cN}^{\frac{4}{3}-r}\left\|s_{0}\right\|_{r, A}^{2},\left\|\eta_{1}^{0}\right\|^{2} \leqslant \mathrm{cN}^{\frac{7}{3}-r}\left\|l_{0}\right\|_{r-2, A}^{2} .
$$

For the estimate $\left\|\bar{\partial}_{t} e_{1}^{0}\right\|$, letting $k=0$ in equation (3.1), setting $v=\bar{\partial}_{t} e_{1}^{0}$ and considering the imaginary part, we obtain

$$
\begin{aligned}
\left\|\bar{\partial}_{t} e_{1}^{0}\right\|^{2} \leqslant & 2 \operatorname{Im}\left(e_{1 x}^{0}, \bar{\partial}_{t} e_{1 x}^{0}\right)_{N}+\alpha \operatorname{Im}\left(\operatorname{I}_{N}\left(s_{N}^{0} l_{N}^{0}\right)-s^{\frac{1}{2}} l^{\frac{1}{2}}, \bar{\partial}_{t} e_{1}^{0}\right)+\operatorname{Re}\left(s_{t}^{\frac{1}{2}}-\bar{\partial}_{t} s^{0}, \bar{\partial}_{t} e_{1}^{0}\right) \\
& \left.+\operatorname{Im}\left(\left(P_{N} s^{0}\right)_{x}, \bar{\partial}_{t} e_{1 x}^{0}\right)_{N}-\left(s_{x}^{\frac{1}{2}}, \bar{\partial}_{t} e_{1 x}^{0}\right)\right)+\operatorname{Im}\left(\left(I_{N}-\mathrm{I}\right) f^{\frac{1}{2}}, \bar{\partial}_{t} e_{1}^{0}\right) \\
\leqslant & \left(c N^{\frac{1}{2}}\left\|e_{1 x}^{0}\right\|_{N}+a_{3}\left(\left\|e_{1}^{0}\right\|+\left\|\eta_{1}^{0}\right\|+\left\|e_{2}^{0}\right\|_{N}+\left\|\eta_{2}^{0}\right\|_{N}+\left\|s^{0}-s^{\frac{1}{2}}\right\|_{2}+\left\|l^{0}-l^{\frac{1}{2}}\right\|+\left\|s_{t}^{\frac{1}{2}}-\bar{\partial}_{t} s^{0}\right\|\right.\right. \\
& \left.+\left\|\left(\left(P_{N}-I\right) s_{x}^{0}\right)_{x}\right\|+c N^{\frac{1}{2}}\left\|\left(\left(P_{N}-I\right) s^{0}\right)_{x}\right\|+\left\|\left(I_{N}-I\right)\left(s^{0} l^{0}\right)\right\|+\left\|\left(I_{N}-I\right) f^{\frac{1}{2}}\right\|\right)\left\|\bar{\partial}_{t} e_{1}^{0}\right\| .
\end{aligned}
$$

By an analogue approach for the estimation of equation (3.10), we infer that

$$
\left\|\bar{\partial}_{\mathrm{t}} e_{1}^{0}\right\|^{2} \leqslant \mathrm{a}_{3}^{2} \max \left\{\mathrm{a}_{4} \mathrm{~T}, c \mathrm{a}_{5} \mathrm{~T}^{2}\right\}\left(\tau^{2}+\mathrm{N}^{\frac{7}{3}-\mathrm{r}}\right) .
$$

Substituting the initial values into equation (3.14) yields

$$
\left\|e_{1}^{n}\right\|_{1, N}^{2}+\left\|\eta_{1}^{n}\right\|^{2} \leqslant a_{15}\left(\tau^{2}+N^{\frac{7}{3}-r}\right)+a_{13} \tau \sum_{k=0}^{n-1}\left(\left\|e_{1}^{k}\right\|_{1, N}^{2}+\left\|\eta_{1}^{k}\right\|^{2}\right),
$$

where $a_{15}=c a_{5}+a_{3}^{2} \max \left\{a_{4}, a_{5}\right\}+a_{14}$. Let $a_{15} \exp \left(a_{13} T\right)\left(\tau^{2}+N^{\frac{7}{3}-r}\right) \leqslant N^{-\frac{1}{2}}$ meet the condition 3 of Lemma 3.2, i.e., $N$ is sufficiently big and $\tau$ is sufficiently small such that $\tau^{2} N^{\frac{1}{2}}$ is sufficiently small and $r \geqslant 3$, then we deduce

$$
\left\|e_{1}^{\mathfrak{n}}\right\|_{1, N}^{2}+\left\|\eta_{1}^{\mathfrak{n}}\right\|^{2} \leqslant a_{15} \exp ^{a_{13} \top}\left(\tau^{2}+N^{\frac{7}{3}-r}\right), \quad 0<n \leqslant M .
$$

Finally, using the triangle inequality and Lemma 2.2, we have

$$
\left\|s^{n}-s_{N}^{n}\right\|_{1, N}+\left\|l^{n}-l_{N}^{n}\right\| \leqslant\left\|e_{2}^{n}\right\|_{1, N}+\left\|e_{1}^{n}\right\|_{1, N}+\left\|\eta_{2}^{n}\right\|+\left\|\eta_{1}^{n}\right\| \leqslant C\left(\tau+N^{\frac{7}{6}-\frac{r}{2}}\right), \quad n=0,1, \cdots, M,
$$

where $C=\sqrt{2 a_{15}} \exp \left(a_{13} T / 2\right)$.

Therefore, we finish the proof of this theorem.

\section{Modified Hermite spectral method for LS equations}

In this section, we first make a priori estimates of discrete solutions then prove the stability and convergence of the scheme (2.10)-(2.13).

Lemma 4.1 ([17]). Assume that $\mathrm{y}_{1} \geqslant 0, \mathrm{~h}_{\mathrm{n}}, \varphi_{\mathrm{n}}$ are non-negative sequences for $\mathrm{n} \geqslant 1$, and $\varphi_{\mathrm{n}}$ satisfies

$$
\left\{\begin{array}{l}
\varphi_{1} \leqslant y_{1}, \\
\varphi_{n} \leqslant y_{1}+\tau \sum_{j=1}^{n-1} h_{j} \varphi_{j}, \quad n \geqslant 2 .
\end{array}\right.
$$

Then it follows

$$
\varphi_{n} \leqslant y_{1} \exp \left(\tau \sum_{j=1}^{n-1} h_{j}\right), n \geqslant 2
$$




\subsection{A priori estimates of the fully discrete scheme}

In what follows, we shall make a priori estimates for equations (2.10)-(2.13) by using the above lemmas.

Lemma 4.2. If $\mathrm{s}_{0} \in \mathrm{H}_{\mathrm{A}}^{3}(\mathbf{R}), \mathrm{l}_{0} \in \mathrm{H}_{\mathrm{A}}^{1}(\mathbf{R}), \mathrm{f} \in \mathrm{L}^{\infty}\left(0, \mathrm{~T} ; \mathrm{H}_{\mathrm{A}}^{1}(\mathbf{R})\right)$, then

$$
\left\|s_{N}^{n}\right\| \leqslant E_{0 s}, \quad n=0,1,2, \cdots, M,
$$

where $\mathrm{E}_{0 \mathrm{~s}}$ is a constant depending on $\mathrm{T}, \mathrm{s}_{0}$ and $\mathrm{f}$.

Proof. Taking $v=s_{N}^{\bar{k}}$ in equation (2.10), and taking the imaginary part, we have

$$
\left\|s_{N}^{k}\right\|_{\hat{\mathrm{t}}} \leqslant\left\|\mathrm{f}^{\mathrm{k}}\right\|_{\mathrm{N}}, \quad k=0,2, \cdots, M-1 .
$$

Using equations (2.12), (2.13), triangle inequality, Theorem 2.3, and Lemma 3.1, we have

$$
\begin{aligned}
\left\|s_{N}^{0}\right\| & \leqslant\left\|I_{N} s_{0}-s_{0}\right\|+\left\|s_{0}\right\| \leqslant c\left\|s_{0}\right\|_{1, A} \\
\left\|s_{N}^{1}\right\| & \leqslant c\left\|s_{0}+i \tau\left(s_{0 x x}-\alpha s_{0} l_{0}-f^{0}\right)\right\|_{1, A} \\
& \leqslant c\left(\left\|s_{0}\right\|_{1, A}+\left\|s_{0 x x}\right\|_{1, A}+\alpha\left\|s_{0}\right\|_{1, A}\left\|l_{0}\right\|_{1, A}+\left\|f^{0}\right\|_{1, A}\right)
\end{aligned}
$$

Thus we infer that

$$
\left\|s_{N}^{n}\right\| \leqslant c\left(\left\|s_{0}\right\|_{1, A}+\left\|s_{0 x x}\right\|_{1, A}+\alpha\left\|s_{0}\right\|_{1, A}\left\|l_{0}\right\|_{1, A}+\|f\|_{L^{\infty}\left(0, T ; H_{A}^{1}(\mathbf{R})\right)}\right) \triangleq E_{0 s}, \quad n=0,1, \cdots, M .
$$

Lemma 4.3. Assume that $s_{0} \in \mathrm{H}_{\AA}^{4}(\mathbf{R}), \mathrm{l}_{0} \in \mathrm{H}_{\AA}^{2}(\mathbf{R}), \mathrm{g} \in \mathrm{L}^{\infty}\left(0, \mathrm{~T} ; \mathrm{H}_{\mathrm{A}}^{1}(\mathbf{R})\right), \mathrm{f} \in \mathrm{L}^{\infty}\left(0, \mathrm{~T} ; \mathrm{H}_{\AA}^{2}(\mathbf{R})\right)$ and $\mathrm{f}_{\mathrm{t}} \in$ $\mathrm{L}^{2}\left(0, \mathrm{~T} ; \mathrm{H}_{\mathrm{A}}^{1}(\mathbf{R})\right)$, then we obtain

$$
\left\|s_{N x}^{n}\right\| \leqslant E_{1 s},\left\|l_{N}^{n}\right\| \leqslant E_{0 l}, \quad n=0,1, \cdots, M, \quad\left\|l_{N \hat{t}}^{n}\right\| \leqslant E_{0 l}^{\prime}, \quad n=1,2, \cdots, M-1,
$$

where $\mathrm{E}_{1 \mathrm{~s}}, \mathrm{E}_{0 \mathrm{l}}$, and $\mathrm{E}_{0 \mathrm{l}}^{\prime}$ are constants depending on $\mathrm{T}, \mathrm{s}_{0}, \mathrm{l}_{0}, \mathrm{f}$, and $\mathrm{g}$.

Proof. First we estimate norms $\left\|l_{N}^{k}\right\|$ and $\left\|l_{N \hat{t}}^{k}\right\|$. Setting $v=l_{N}^{\bar{k}}$ in equation (2.11), then using Sobolev inequality $\left\|s_{N}^{k}\right\|_{\infty} \leqslant\left\|s_{N}^{k}\right\| \frac{1}{2}\left\|s_{N x}^{k}\right\|^{\frac{1}{2}}$, the property $\left\|s_{N x}^{k}\right\|_{N} \leqslant\left\|s_{N x}^{k}\right\|$ due to $I_{N} s_{N+1}^{k}=P_{N} s_{N+1}^{k}$ and Lemma 4.2, we obtain

$$
\frac{1}{2}\left\|l_{N}^{k}\right\|_{\hat{\mathrm{t}}}^{2} \leqslant 2 \beta\left\|s_{N}^{k}\right\|^{\frac{1}{2}}\left\|s_{N x}^{k}\right\|^{\frac{3}{2}}\left\|l_{N}^{\bar{k}}\right\|+\left\|g^{k}\right\|_{N}\left\|l_{N}^{\bar{k}}\right\| \leqslant\left(2 \beta E_{0 s}^{\frac{1}{2}}\left\|s_{N x}^{k}\right\|^{\frac{3}{2}}+\left\|g^{k}\right\|_{N}\right)\left\|l_{N}^{\bar{k}}\right\|
$$

It further gives,

$$
\left\|l_{N}^{k+1}\right\|-\left\|l_{N}^{k-1}\right\| \leqslant 4 \beta E_{0 s}^{\frac{1}{2}} \tau\left\|s_{N x}^{k}\right\|^{\frac{3}{2}}+2 \tau\left\|g^{k}\right\|_{N} .
$$

Taking the sum for $k$ from 1 to $n-1$, we infer that

$$
\left\|l_{N}^{n}\right\|+\left\|l_{N}^{n-1}\right\| \leqslant\left\|l_{N}^{0}\right\|+\left\|l_{N}^{1}\right\|+c T \max _{t}\|g\|_{1, A}+4 \beta E_{0 s}^{\frac{1}{2}} \tau \sum_{k=1}^{n-1}\left\|s_{N x}^{k}\right\|^{\frac{3}{2}}, \quad n=2,3, \cdots, M
$$

Using equation (2.11), Lemma 4.2 and Theorem 2.3, we deduce that

$$
\left\|l_{N \hat{t}}^{k}\right\| \leqslant 2 \beta\left\|s_{N}^{k}\right\|^{\frac{1}{2}}\left\|s_{N x}^{k}\right\|^{\frac{3}{2}}+\left\|g^{k}\right\|_{N} \leqslant 2 \beta E_{0 s}^{\frac{1}{2}}\left\|s_{N x}^{k}\right\|^{\frac{3}{2}}+c \max _{t}\|g\|_{1, A,} \quad k=1,2, \cdots, M-1 .
$$

Next we estimate $\left\|s_{N x}^{k}\right\|$. To the end, setting $v=s_{N \hat{t}}^{k}$ in equation (2.10) and taking the real part, then 
taking the sum for $k$ from 1 to $n-1$, using the identity

$$
2 \tau \sum_{k=1}^{n-1}\left(u^{k}, v_{\hat{t}}^{k}\right)=-2 \tau \sum_{k=1}^{n-1}\left(u_{\hat{\mathrm{t}}}^{k}, v^{k}\right)+\left(u^{n}, v^{n-1}\right)+\left(u^{n-1}, v^{n}\right)-\left(u^{1}, v^{0}\right)-\left(u^{0}, v^{1}\right),
$$

we derive that

$$
\begin{aligned}
\left\|s_{N x}^{n}\right\|^{2}+\left\|s_{N x}^{n-1}\right\|^{2}-\left\|s_{N x}^{0}\right\|^{2}-\left\|s_{N x}^{1}\right\|^{2}= & 4 \tau \sum_{k=1}^{n-1} \operatorname{Re}\left(f^{k}, s_{N \hat{t}}^{k}\right)_{N}-2 \alpha \tau \sum_{k=1}^{n-1}\left(l_{N}^{k},\left|s_{N}^{k}\right|_{\hat{t}}^{2}\right)_{N} \\
= & -4 \tau \sum_{k=1}^{n-1} \operatorname{Re}\left(f_{\hat{t}}^{k}, s_{N}^{k}\right)_{N}+2\left(\operatorname{Re}\left(f^{n}, s_{N}^{n-1}\right)_{N}+\operatorname{Re}\left(f^{n-1}, s_{N}^{n}\right)_{N}\right. \\
& \left.-\operatorname{Re}\left(f^{1}, s_{N}^{0}\right)_{N}-\operatorname{Re}\left(f^{0}, s_{N}^{1}\right)_{N}\right) \\
& +2 \alpha \tau \sum_{k=1}^{n-1}\left(l_{N \hat{t},}^{k}\left|s_{N}^{k}\right|^{2}\right)_{N}-\alpha\left(\left(l_{N}^{n},\left|s_{N}^{n-1}\right|^{2}\right)_{N}+\left(l_{N}^{n-1},\left|s_{N}^{n}\right|^{2}\right)_{N}\right. \\
& \left.-\left(l_{N}^{1},\left|s_{N}^{0}\right|^{2}\right)_{N}-\left(l_{N}^{0},\left|s_{N}^{1}\right|^{2}\right)_{N}\right) .
\end{aligned}
$$

Using Hölder inequality, Lemma 4.2 and Theorem 2.3, we obtain

$$
\begin{aligned}
-4 \tau \sum_{k=1}^{n-1} \operatorname{Re}\left(f_{\hat{\mathrm{t}}}^{k}, s_{N}^{k}\right)_{N} & \leqslant 4 \tau \sum_{k=1}^{n-1}\left\|\frac{1}{2 \tau} \int_{t_{k-1}}^{t_{k+1}} f_{t} d t\right\|_{N}\left\|s_{N}^{k}\right\| \leqslant c T^{\frac{1}{2}} E_{0 s}\left(\int_{0}^{T}\left\|f_{t}\right\|_{1, A}^{2} d t\right)^{\frac{1}{2}}, \\
2 \operatorname{Re}\left(f^{k_{1}}, s_{N}^{k_{2}}\right)_{N} & \leqslant c E_{0 s} \max _{t}\|f\|_{1, A},\left(k_{1}, k_{2}\right)=(n, n-1),(n-1, n),(1,0) \text { or }(0,1) .
\end{aligned}
$$

According to equation (4.2), we have

$$
2 \alpha \tau \sum_{k=1}^{n-1}\left(l_{N \hat{t},}^{k}\left|s_{N}^{k}\right|^{2}\right)_{N} \leqslant c \alpha T E_{0 s}^{\frac{3}{2}} \max _{t}\|g\|_{1, A}^{\frac{4}{3}}+\left(4 \alpha \beta E_{0 s}^{2}+\frac{1}{2} \alpha E_{0 s}^{\frac{3}{2}}\right) \tau \sum_{k=1}^{n-1}\left\|s_{N x}^{k}\right\|^{2} .
$$

Using equation (4.1) and Sobolev inequality for $\left(k_{1}, k_{2}\right)=(n, n-1)$ or $(n-1, n)$, we deduce

$$
\begin{aligned}
\alpha\left(l_{N}^{k_{1}},\left|s_{N}^{k_{2}}\right|^{2}\right)_{N} \leqslant & \alpha E_{0 s}^{\frac{3}{2}}\left\|s_{N x}^{k_{2}}\right\| \|^{\frac{1}{2}}\left(\left\|l_{N}^{0}\right\|+\left\|l_{N}^{1}\right\|+c T \max _{t}\|g\|_{1, A}+4 \beta E_{0 s}^{\frac{1}{2}} \tau \sum_{k=1}^{k_{1}-1}\left\|s_{N x}^{k}\right\|^{\frac{3}{2}}\right) \\
\leqslant & \frac{1}{2}\left\|s_{N x}^{k_{2}}\right\|^{2}+\frac{3}{4} \alpha^{\frac{4}{3}} E_{0 s}^{2}\left(\left\|l_{N}^{0}\right\|+\left\|l_{N}^{1}\right\|+c T \max _{t}\|g\|_{1, A}\right)^{\frac{4}{3}} \\
& +\frac{3}{4} T^{\frac{1}{3}}\left(4 \alpha \beta E_{0 s}^{2}\right)^{\frac{4}{3}} \tau \sum_{k=1}^{k_{1}-1}\left\|s_{N x}^{k}\right\|^{2} \\
\alpha\left(l_{N}^{1},\left|s_{N}^{0}\right|^{2}\right)_{N}+\alpha\left(l_{N}^{0},\left|s_{N}^{1}\right|^{2}\right)_{N} \leqslant & \frac{1}{4}\left(\left\|s_{N x}^{0}\right\|^{2}+\left\|s_{N x}^{1}\right\|^{2}\right)+\frac{3}{4} \alpha^{\frac{4}{3}} E_{0 s}^{2}\left(\left\|l_{N}^{0}\right\|^{\frac{4}{3}}+\left\|l_{N}^{1}\right\|^{\frac{4}{3}}\right) .
\end{aligned}
$$

Substituting all the above estimates into equation (4.4) yields

$$
\left\|s_{N x}^{n}\right\|^{2} \leqslant y_{1}+h \tau \sum_{k=1}^{n-1}\left\|s_{N x}^{k}\right\|^{2}, \quad n=2,3, \cdots, M,
$$

where

$$
y_{1}=\frac{5}{2}\left(\left\|s_{N x}^{0}\right\|^{2}+\left\|s_{N x}^{1}\right\|^{2}\right)+c T^{\frac{1}{2}} E_{0 s}\left(\int_{0}^{T}\left\|f_{t}\right\|_{1, A}^{2} d t\right)^{\frac{1}{2}}+c E_{0 s} \max _{t}\|f\|_{1, A}
$$




$$
\begin{aligned}
& +c \alpha \mathrm{TE}_{0 s}^{\frac{3}{2}} \max _{t}\|g\|_{1, A}^{\frac{4}{3}}+3 \alpha^{\frac{4}{3}} \mathrm{E}_{0 s}^{2}\left(\left\|l_{N}^{0}\right\|+\left\|l_{N}^{1}\right\|+c T \max _{t}\|g\|_{1, A}\right)^{\frac{4}{3}}, \\
h= & 8 \alpha \beta \mathrm{E}_{0 s}^{2}+\alpha \mathrm{E}_{0 s}^{\frac{3}{2}}+3 T^{\frac{1}{3}}\left(4 \alpha \beta \mathrm{E}_{0 s}^{2}\right)^{\frac{4}{3}} .
\end{aligned}
$$

It is obvious to see that $\left\|s_{N_{x}}^{1}\right\|^{2} \leqslant y_{1}$. For initial values, using equations (2.12), (2.13), and Lemma 3.1, Theorem 2.3, we obtain

$$
\begin{aligned}
\left\|s_{N x}^{0}\right\| & \leqslant\left\|\left(I_{N} s_{0}-s_{0}\right)_{x}\right\|+\left\|s_{0 x}\right\| \leqslant c\left\|s_{0}\right\|_{2, A},\left\|l_{N}^{0}\right\| \leqslant c\left\|l_{0}\right\|_{1, A}, \\
\left\|s_{N x}^{1}\right\| & \leqslant c\left(\left\|s_{0}\right\|_{2, A}+\left\|s_{0 x x}\right\|_{2, A}+\alpha\left\|s_{0}\right\|_{2, A}\left\|l_{0}\right\|_{2, A}+\max _{t}\|f\|_{2, A}\right) \triangleq b_{1}, \\
\left\|l_{N}^{1}\right\| & \leqslant c\left(\left\|l_{0}\right\|_{2, A}+\beta\left\|s_{0}\right\|_{1, A}\left\|s_{0 x}\right\|_{1, A}+\max _{t}\|g\|_{1, A}\right) \triangleq b_{2} .
\end{aligned}
$$

Now applying Lemma 4.1 for equation (4.5) leads to

$$
\left\|s_{N x}^{n}\right\|^{2} \leqslant y \exp (h T) \triangleq E_{1 s}^{2}, \quad n=2,3, \cdots, M,
$$

where

$$
\begin{aligned}
y= & c\left\|s_{0}\right\|_{2, A}+b_{1}+c T^{\frac{1}{2}} E_{0 s}\left(\int_{0}^{T}\left\|f_{t}\right\|_{1, A}^{2} d t\right)^{\frac{1}{2}}+c E_{0 s} \max _{t}\|f\|_{1, A}+c \alpha T E_{0 s}^{\frac{3}{2}} \max _{t}\|g\|_{1, A}^{\frac{4}{3}} \\
& +3 \alpha^{\frac{4}{3}} E_{0 s}^{2}\left(c\left\|l_{0}\right\|_{1, A}+b_{2}+c T \max _{t}\|g\|_{1, A}\right)^{\frac{4}{3}} .
\end{aligned}
$$

Substituting equation (4.7) into equations (4.1) and (4.2), respectively, we obtain

$$
\left\|l_{N}^{n}\right\| \leqslant c\left\|l_{0}\right\|_{1, A}+b_{2}+c T \max _{t}\|g\|_{1, A}+4 \beta E_{0 s}^{\frac{1}{2}} T_{1 s}^{\frac{3}{2}} \triangleq E_{0 l}, \quad n=2,3, \cdots, M
$$

and

$$
\left\|l_{\mathrm{N} \hat{\mathrm{t}}}^{n}\right\| \leqslant c \max _{\mathrm{t}}\|g\|_{1, A}+2 \beta \mathrm{E}_{0 \mathrm{~s}}^{\frac{1}{2}} \mathrm{E}_{1 \mathrm{~s}}^{\frac{3}{2}} \triangleq \mathrm{E}_{0 l}^{\prime}, \quad \mathrm{n}=1,2, \cdots, M-1 .
$$

Consequently, the proof of Lemma 4.3 is accomplished.

Lemma 4.4. If $\mathrm{s}_{0} \in \mathrm{H}_{\mathrm{A}}^{5}(\mathbf{R}), \mathrm{l}_{0} \in \mathrm{H}_{\mathrm{A}}^{3}(\mathbf{R}), \mathrm{g} \in \mathrm{L}^{\infty}\left(0, \mathrm{~T} ; \mathrm{H}_{\mathrm{A}}^{1}(\mathbf{R})\right), \mathrm{f} \in \mathrm{L}^{\infty}\left(0, \mathrm{~T} ; \mathrm{H}_{\mathrm{A}}^{3}(\mathbf{R})\right)$, and $\mathrm{f}_{\mathrm{t}} \in \mathrm{L}^{2}\left(0, \mathrm{~T} ; \mathrm{H}_{A}^{1}(\mathbf{R})\right)$, we obtain

$$
\left\|s_{N \hat{t}}^{n}\right\| \leqslant E_{0 s}^{\prime}, \quad n=1,2, \cdots, M-1,
$$

where $\mathrm{E}_{0 \mathrm{~s}}^{\prime}$ is a constant depending on $\mathrm{T}, \mathrm{s}_{0}, \mathrm{l}_{0}, \mathrm{f}$, and $\mathrm{g}$.

Proof. Setting $w_{\mathrm{N}}^{k}=s_{\mathrm{N} \hat{\mathrm{t}}}^{\mathrm{k}}$, then making central difference quotient for equation (2.10), we obtain

$$
\mathrm{i}\left(w_{\mathrm{N} \hat{\mathrm{t}}}^{\mathrm{k}}, v\right)+\left(w_{\mathrm{N} x}^{\bar{k}}, v_{x}\right)=\frac{\alpha}{2 \tau}\left(s_{N}^{\bar{k}+1} l_{N}^{k+1}-s_{N}^{\bar{k}} l_{N}^{k}, v\right)_{N}+\left(f_{\hat{\mathrm{t}}}^{k}, v\right)_{N}, \quad k=1,2, \cdots, M-2
$$

Setting $v=w_{\mathrm{N}}^{\overline{\mathrm{k}}}$ in equation (4.8), taking the imaginary part, using Hölder inequality, Lemmas 4.2 and 4.3, one has

$$
\frac{1}{2}\left\|w_{N}^{k}\right\|_{\hat{\mathrm{t}}}^{2}=\operatorname{Im}\left(\alpha s_{N}^{\bar{k}} l_{N \hat{t}}^{k}+f_{\hat{\mathrm{t}}}^{k}, w_{\mathrm{N}}^{\bar{k}}\right)_{N} \leqslant\left(\alpha E_{0 s}^{\frac{1}{2}} E_{1 s}^{\frac{1}{2}} E_{0 l}^{\prime}+\left\|f_{\hat{\mathrm{t}}}^{k}\right\|_{N}\right)\left\|w_{N}^{\bar{k}}\right\|
$$

It further gives,

$$
\left\|w_{\mathrm{N}}^{k+1}\right\|-\left\|w_{\mathrm{N}}^{k-1}\right\| \leqslant 2 \tau\left(\alpha \mathrm{E}_{0 \mathrm{~s}}^{\frac{1}{2}} \mathrm{E}_{1 \mathrm{~s}}^{\frac{1}{2}} \mathrm{E}_{0 \mathrm{l}}^{\prime}+\left\|\mathrm{f}_{\hat{\mathrm{t}}}^{\mathrm{k}}\right\|_{\mathrm{N}}\right) .
$$

Taking the sum for $k$ from 2 to $n-2$ and using Hölder inequality, we infer that

$$
\left\|w_{N}^{n-1}\right\|^{2}+\left\|w_{N}^{n-2}\right\|^{2} \leqslant 4\left(\left\|w_{N}^{1}\right\|^{2}+\left\|w_{N}^{2}\right\|^{2}+4 \alpha^{2} T^{2} E_{0 s} E_{1 s} E_{0 l}^{\prime 2}+c T \int_{0}^{T}\left\|f_{t}\right\|_{1, A}^{2} d t\right)
$$

Now we estimate $\left\|w_{N}^{1}\right\|$ and $\left\|w_{N}^{2}\right\|$, in other words $\left\|s_{N \hat{t}}^{1}\right\|$ and $\left\|s_{N \hat{t}}^{2}\right\|$. Letting $k=1$ in equation (2.10) then 
setting $v=s_{\mathrm{N} \hat{\mathrm{t}}}^{1}$ in and taking the imaginary part, using equation (4.6) and Theorem 2.3, it follows

$$
\begin{aligned}
\left\|s_{N \hat{t}}^{1}\right\|^{2} & =\operatorname{Im}\left(s_{N x x}^{0}, s_{N \hat{t}}^{1}\right)+\alpha \operatorname{Im}\left(l_{N}^{1} s_{N}^{0}, s_{N \hat{t}}^{1}\right)_{N}+\operatorname{Im}\left(f^{1}, s_{N \hat{t}}^{1}\right)_{N} \\
& \leqslant c\left(\left\|s_{0}\right\|_{3, A}+\alpha b_{2}\left\|s_{0}\right\|_{1, A}^{\frac{1}{2}}\left\|s_{0}\right\|_{2, A}^{\frac{1}{2}}+\max _{t}\|f\|_{1, A}\right)\left\|s_{N \hat{t}}^{1}\right\| .
\end{aligned}
$$

Namely,

$$
\left\|s_{\mathrm{N} \hat{\mathrm{t}}}^{1}\right\| \leqslant \mathrm{c}\left(\left\|s_{0}\right\|_{3, \mathrm{~A}}+\alpha \mathrm{b}_{2}\left\|s_{0}\right\|_{1, \mathrm{~A}}^{\frac{1}{2}}\left\|s_{0}\right\|_{2, \mathrm{~A}}^{\frac{1}{2}}+\max _{\mathrm{t}}\|f\|_{1, A}\right) \triangleq \mathrm{b}_{3} .
$$

Similarly to equation (4.10), setting $k=2$ in equation (2.10) and using Lemmas 4.2, 4.3, and Theorem 2.3, we have

$$
\begin{aligned}
\left\|s_{N \hat{t}}^{2}\right\|^{2} & =\operatorname{Im}\left(s_{N x x}^{1}, s_{N \hat{t}}^{2}\right)+\operatorname{Im}\left(\operatorname{I}_{N}\left(l_{N}^{2} s_{N}^{1}\right), s_{N \hat{t}}^{2}\right)+\operatorname{Im}\left(f^{2}, s_{N \hat{t}}^{2}\right){ }_{N} \\
& \leqslant c\left(\left\|s_{0}\right\|_{3, A}+\left\|s_{0 x x}\right\|_{3, A}+\alpha\left\|s_{0}\right\|_{3, A}\left\|l_{0}\right\|_{3, A}+\alpha b_{1}^{\frac{1}{2}} E_{0 s}^{\frac{1}{2}} E_{0 l}+\max _{t}\|f\|_{3, A}\right)\left\|s_{N \hat{t}}^{2}\right\| .
\end{aligned}
$$

That is,

$$
\left\|s_{N \hat{t}}^{2}\right\| \leqslant c\left(\left\|s_{0}\right\|_{3, A}+\left\|s_{0 x x}\right\|_{3, A}+\alpha\left\|s_{0}\right\|_{3, A}\left\|l_{0}\right\|_{3, A}+\alpha b_{1}^{\frac{1}{2}} E_{0 s}^{\frac{1}{2}} E_{0 l}+\max _{t}\|f\|_{3, A}\right) \triangleq b_{4} .
$$

Substituting equations (4.10) and (4.11) into (4.9), we obtain

$$
\left\|w_{N}^{\mathfrak{n}}\right\|^{2} \leqslant 4\left(b_{3}^{2}+b_{4}^{2}+4 \alpha^{2} T^{2} E_{0 s} E_{1 s} E_{0 l}^{\prime 2}+c T \int_{0}^{T}\left\|f_{t}\right\|_{1, A}^{2} d t\right) \triangleq E_{0 s}^{\prime 2} .
$$

Finally, we finish the proof of Lemma 4.4.

\subsection{Numerical stability of the fully discrete scheme}

The main purpose in this section is to prove the unconditional stability for the discrete scheme (2.10)(2.13).

Suppose that $s_{N j}^{k}$ and $l_{N j}^{k}$ are two solutions of equations (2.10)-(2.13) with the initial values $s_{N j}^{0}, s_{N j}^{1}$, $l_{N j}^{0}, l_{N j}^{1}$ and the source terms $f_{j}, g_{j}(j=1,2)$, respectively. We can obtain the following results as proved in Subsection 4.1:

$$
\left\|s_{N j}^{k}\right\| \leqslant E_{0 s j}, \quad\left\|s_{N j x}^{k}\right\| \leqslant E_{1 s j}, \quad\left\|s_{N j \hat{t}}^{k}\right\| \leqslant E_{0 s j}^{\prime}, j=1,2
$$

and

$$
\left\|l_{N j}^{k}\right\| \leqslant E_{0 l j}, \quad\left\|l_{N j \hat{t}}^{k}\right\| \leqslant E_{0 l j}^{\prime}, j=1,2,
$$

where $E_{0 s j}, E_{1 s j}$ and $E_{0 l j}$ depend on $s_{N j}^{0}, s_{N j}^{1}, l_{N j}^{0}, l_{N j}^{1}, f_{j}$ and $g_{j}(j=1,2)$.

Setting $u_{N}^{k}=s_{N 1}^{k}-s_{N 2}^{k}, v_{N}^{k}=l_{N 1}^{k}-l_{N 2}^{k}, \tilde{f}^{k}=f_{1}^{k}-f_{2}^{k}$, and $\tilde{g}^{k}=g_{1}^{k}-g_{2}^{k}$, we can find that $u_{N}^{k}$ and $v_{N}^{k}$ satisfy the following equations

$$
\left\{\begin{array}{l}
i\left(u_{N \hat{t}}^{k}, v\right)+\left(u_{N x}^{\bar{k}}, v_{x}\right)=\alpha\left(s_{N 1}^{\bar{k}} l_{N 1}^{k}-s_{N 2}^{\bar{k}} l_{N 2}^{k}, v\right)_{N}+\left(\tilde{f}^{k}, v\right)_{N}, \quad k=1,2, \cdots, M-1, \\
\left(v_{N \hat{t}}^{k}, v\right)+\beta\left(\left(\left|s_{N 1}^{k}\right|^{2}\right)_{x}-\left(\left|s_{N 2}^{k}\right|^{2}\right)_{x}, v\right)_{N}=\left(\tilde{g}^{k}, v\right)_{N}, \quad k=1,2, \cdots, M-1 .
\end{array}\right.
$$

The following result is regarding the stability of the fully discrete scheme (2.10)-(2.13).

Theorem 4.5. Assume that initial values and source terms satisfy the conditions of Lemmas 4.2-4.4, then the fully discrete scheme (2.10)-(2.13) is unconditionally stable

$$
\begin{aligned}
\left\|u_{N}^{n}\right\|_{1}^{2}+\left\|v_{N}^{n}\right\|^{2} \leqslant & C\left(\left\|u_{N}^{0}\right\|_{1}^{2}+\left\|u_{N}^{1}\right\|_{1}^{2}+\left\|v_{N}^{0}\right\|^{2}+\left\|v_{N}^{1}\right\|^{2}+\|\tilde{g}\|_{L^{\infty}\left(0, T ; H^{1}(\mathbf{R})\right)}^{2}\right. \\
& \left.+\|\tilde{f}\|_{L^{\infty}\left(0, T ; H^{1}(\mathbf{R})\right)}^{2}+\int_{0}^{T}\left\|\tilde{f_{t}}\right\|_{1, A}^{2} d t\right), \quad n=1,2, \cdots, M
\end{aligned}
$$

where the constant $C$ is independent of $\tau$ and $\mathrm{N}$. 
Proof. Setting $v=u_{N}^{\bar{k}}$ in equation (4.14), taking the imaginary part, then using Hölder inequality and equation (4.12), we have

$$
\frac{1}{2}\left\|u_{N}^{k}\right\|_{\hat{t}}^{2}=\alpha \operatorname{Im}\left(s_{N 1}^{\bar{k}} v_{N}^{k}, u_{N}^{\bar{k}}\right)_{N}+\operatorname{Im}\left(f_{N}^{k}, u_{N}^{\bar{k}}\right)_{N} \leqslant\left(\alpha E_{0 s 1}^{\frac{1}{2}} E_{1 s 1}^{\frac{1}{2}}\left\|v_{N}^{k}\right\|+\left\|\tilde{f}^{k}\right\|_{N}\right)\left\|u_{N}^{\bar{k}}\right\| .
$$

After simplification,

$$
\left\|u_{N}^{k}\right\|_{\hat{\mathrm{t}}} \leqslant \alpha \mathrm{E}_{0 \mathrm{~s} 1}^{\frac{1}{2}} \mathrm{E}_{1 \mathrm{~s} 1}^{\frac{1}{2}}\left\|v_{\mathrm{N}}^{\mathrm{k}}\right\|+\left\|\tilde{f}^{k}\right\|_{N} .
$$

Taking the sum for $\mathrm{k}$ from 1 to $\mathrm{n}-1$, we infer that

$$
\left\|u_{N}^{n}\right\|+\left\|u_{N}^{n-1}\right\| \leqslant\left\|u_{N}^{0}\right\|+\left\|u_{N}^{1}\right\|+c T \max _{t}\|\tilde{f}\|_{1, A}+2 \alpha E_{0 s 1}^{\frac{1}{2}} E_{1 s 1}^{\frac{1}{2}} \tau \sum_{k=0}^{n-1}\left\|v_{N}^{k}\right\| .
$$

Now we estimate $\left\|v_{N}^{k}\right\|$. Taking $v=v_{N}^{\bar{k}}$ in equation (4.15), using Hölder inequality, Young's inequality, $\left\|u_{N x}\right\|_{N} \leqslant\left\|u_{N x}\right\|$ due to $I_{N} u_{N+1}=P_{N} u_{N+1}$ and equation (4.12), we deduce that

$$
\begin{aligned}
\frac{1}{2}\left\|v_{N}^{k}\right\|_{\hat{\mathrm{t}}}^{2} & \leqslant 2 \beta\left(\left\|\bar{s}_{\mathrm{N} 1}^{\mathrm{k}}\right\|_{\infty}\left\|u_{N x}^{k}\right\|+\left\|s_{N 2 x}^{k}\right\|\left\|u_{N}^{k}\right\|^{\frac{1}{2}}\left\|u_{N x}^{k}\right\| \frac{1}{2}\right)\left\|v_{N}^{\bar{k}}\right\|+\left\|\tilde{g}^{k}\right\|_{N}\left\|v_{N}^{\bar{k}}\right\| \\
& \leqslant \beta b_{5}\left(\left\|u_{N x}^{k}\right\|+\left\|u_{N}^{k}\right\|\right)\left\|v_{N}^{\bar{k}}\right\|+\left\|\tilde{g}^{k}\right\|_{N}\left\|v_{N}^{\bar{k}}\right\| .
\end{aligned}
$$

That is,

$$
\left\|v_{N}^{k}\right\|_{\hat{t}} \leqslant \beta b_{5}\left(\left\|u_{N x}^{k}\right\|+\left\|u_{N}^{k}\right\|\right)+\left\|\tilde{g}^{k}\right\|_{N}
$$

where $b_{5}=2 E_{0 s 1}^{\frac{1}{2}} E_{1 s 1}^{\frac{1}{2}}+E_{1 s 2}$. Evaluating the sum for $k$ from 1 to $n-1$, we deduce

$$
\left\|v_{N}^{n}\right\|+\left\|v_{N}^{n-1}\right\| \leqslant\left\|v_{N}^{0}\right\|+\left\|v_{N}^{1}\right\|+c T \max _{t}\|\tilde{g}\|_{1, A}+2 \beta b_{5} \tau \sum_{k=0}^{n-1}\left(\left\|u_{N}^{k}\right\|+\left\|u_{N x}^{k}\right\|\right) .
$$

Now we estimate $\left\|u_{N x}^{k}\right\|$ on the right hand of equation (4.17). Setting $v=u_{N \hat{t}}^{k}$ in equation (4.14) and considering the real part, then taking the sum for $\mathrm{k}$ from 1 to $\mathrm{n}-1$, we obtain

$$
\begin{aligned}
& \left\|u_{N x}^{n}\right\|^{2}+\left\|u_{N x}^{n-1}\right\|^{2}-\left\|u_{N x}^{0}\right\|^{2}-\left\|u_{N x}^{1}\right\|^{2} \\
& =-4 \alpha \tau \operatorname{Re} \sum_{k=1}^{n-1}\left(s_{N 1}^{\bar{k}} v_{N}^{k}, u_{N \hat{t}}^{k}\right)_{N}-2 \alpha \tau \sum_{k=1}^{n-1}\left(l_{N 2}^{k},\left|u_{N}^{k}\right|_{\hat{t}}^{2}\right)_{N}-4 \tau \operatorname{Re} \sum_{k=1}^{n-1}\left(\tilde{f}^{k}, u_{N \hat{t}}^{k}\right)_{N} .
\end{aligned}
$$

Now we estimate the terms on the right hand side of equation (4.18), respectively. For the first term, using equation (4.3), Hölder inequality, equation (4.12) and $\left\|v_{N \hat{t}}^{k}\right\| \leqslant \beta b_{5}\left(\left\|u_{N x}^{k}\right\|+\left\|u_{N}^{k}\right\|\right)+\left\|\tilde{g}^{k}\right\|_{N}$, we infer that

$$
\begin{aligned}
-4 \alpha \tau \operatorname{Re} \sum_{k=1}^{n-1}\left(s_{N 1}^{\bar{k}} v_{N}^{k}, u_{N \hat{t}}^{k}\right)_{N}= & 4 \alpha \tau \operatorname{Re} \sum_{k=1}^{n-2}\left(\left(s_{N 1}^{\bar{k}} v_{N}^{k}\right)_{\hat{t}}, u_{N}^{k}\right)_{N}-2 \alpha \operatorname{Re}\left(\left(s_{N 1}^{\bar{n}-2} v_{N}^{n-2}, u_{N}^{n-1}\right)_{N}\right. \\
& \left.+\left(s_{N 1}^{\bar{n}-1} v_{N}^{n-1}, u_{N}^{n}\right)_{N}-\left(s_{N 1}^{\overline{0}} v_{N}^{0}, u_{N}^{1}\right)_{N}-\left(s_{N 1}^{\overline{1}} v_{N}^{1}, u_{N}^{0}\right)_{N}\right) \\
\leqslant & 4 \alpha \tau \sum_{k=1}^{n-2}\left(E_{0 s 1}^{\prime}\left\|v_{N}^{k+1}\right\|\left\|u_{N}^{k}\right\| \frac{1}{2}\left\|u_{N x}^{k}\right\|^{\frac{1}{2}}+E_{0 s 1}^{\frac{1}{2}} E_{1 s 1}^{\frac{1}{2}}\left\|v_{N \hat{t}}^{k}\right\|\left\|u_{N}^{k}\right\|\right) \\
& +2 \alpha E_{0 s 1}^{\frac{1}{2}} E_{1 s 1}^{\frac{1}{2}}\left(\left\|v_{N}^{n-2}\right\|\left\|u_{N}^{n-1}\right\|+\left\|v_{N}^{n-1}\right\|\left\|u_{N}^{n}\right\|+\left\|v_{N}^{0}\right\|\left\|u_{N}^{1}\right\|+\left\|v_{N}^{1}\right\|\left\|u_{N}^{0}\right\|\right) \\
\leqslant & \alpha E_{0 s 1}^{\frac{1}{2}} E_{1 s 1}^{\frac{1}{2}}\left(c T \max _{t}\|\tilde{g}\|_{1, A}^{2}+\left\|u_{N}^{0}\right\|^{2}+\left\|u_{N}^{1}\right\|^{2}+\left\|v_{N}^{0}\right\|^{2}+\left\|v_{N}^{1}\right\|^{2}\right) \\
& +b_{6} \tau \sum_{k=1}^{n-2}\left(\left\|v_{N}^{k+1}\right\|^{2}+\left\|u_{N}^{k}\right\|_{1}^{2}\right) \\
& +\alpha E_{0 s 1}^{\frac{1}{2}} E_{1 s 1}^{\frac{1}{2}}\left(\left\|v_{N}^{n-1}\right\|^{2}+\left\|v_{N}^{n-2}\right\|^{2}+\left\|u_{N}^{n-1}\right\|^{2}+\left\|u_{N}^{n}\right\|^{2}\right),
\end{aligned}
$$


where $b_{6}=2 \alpha\left(E_{0 s 1}^{\prime}+E_{0 s 1}^{\frac{1}{2}} E_{1 s 1}^{\frac{1}{2}}\left(5 \beta b_{5}+2\right)\right)$. Similarly, using equation (4.13), we have

$$
\begin{aligned}
-2 \alpha \tau \sum_{k=1}^{n-1}\left(l_{N 2}^{k},\left|u_{N}^{k}\right|_{\hat{\mathrm{t}}}^{2}\right)_{N} \leqslant & \frac{3}{4} \mathrm{E}_{0 \mathrm{l} 2}^{\frac{4}{3}} \alpha^{\frac{4}{3}}\left(\left\|u_{N}^{1}\right\|^{2}+\left\|u_{N}^{0}\right\|^{2}\right)+\frac{1}{4}\left(\left\|u_{N x}^{1}\right\|^{2}+\left\|u_{N x}^{0}\right\|^{2}\right) \\
& +\frac{3}{2} \alpha E_{012}^{\prime} \tau \sum_{k=2}^{n-2}\left\|u_{N}^{k}\right\|_{1}^{2}+\frac{3}{4} E_{0 l 2}^{\frac{4}{3}} \alpha^{\frac{4}{3}}\left(\left\|u_{N}^{n-1}\right\|^{2}+\left\|u_{N}^{n}\right\|^{2}\right)+\frac{1}{4}\left(\left\|u_{N x}^{n}\right\|^{2}+\left\|u_{N x}^{n-1}\right\|^{2}\right) \\
-4 \tau \operatorname{Re} \sum_{k=1}^{n-1}\left(\tilde{f}^{k}, u_{N \hat{t}}^{k}\right)_{N} \leqslant & \left\|u_{N}^{1}\right\|^{2}+\left\|u_{N}^{0}\right\|^{2}+c \max _{t}\|\tilde{f}\|_{1, A}^{2}+c \int_{0}^{T}\left\|\tilde{f}_{t}\right\|_{1, A}^{2} d t+2 \tau \sum_{k=1}^{n-1}\left\|u_{N}^{k}\right\|^{2} \\
& +\left\|u_{N}^{n}\right\|^{2}+\left\|u_{N}^{n-1}\right\|^{2} .
\end{aligned}
$$

Substituting the above three estimates into equation (4.18) and using equations (4.16) and (4.17) leads to

$$
\begin{aligned}
\left\|u_{N x}^{n}\right\|^{2} \leqslant & +b_{7}\left(\left\|u_{N}^{0}\right\|_{1}^{2}+\left\|v_{N}^{0}\right\|^{2}+\left\|u_{N}^{1}\right\|_{1}^{2}+\left\|v_{N}^{1}\right\|^{2}+\max _{t}\left(\|\tilde{f}\|_{1, A}^{2}+\|\tilde{g}\|_{1, A}^{2}\right)+\int_{0}^{T}\left\|\tilde{f}_{t}\right\|_{1, A}^{2} d t\right) \\
& +b_{8} \tau \sum_{k=1}^{n-1}\left(\left\|v_{N}^{k}\right\|^{2}+\left\|u_{N}^{k}\right\|_{1}^{2}\right)
\end{aligned}
$$

where

$$
\begin{aligned}
& \mathrm{b}_{7}=5 \alpha^{\frac{4}{3}} \mathrm{E}_{0 \mathrm{l} 2}^{\frac{4}{3}}+\mathrm{c} \alpha \mathrm{E}_{0 \mathrm{~s} 1}^{\frac{1}{2}} \mathrm{E}_{1 \mathrm{~s} 1}^{\frac{1}{2}} \mathrm{~T}+\frac{20}{3} \\
& \mathrm{~b}_{8}=\frac{4}{3} \mathrm{~b}_{6}+\max \left\{2 \alpha \mathrm{E}_{012}^{\prime}+\frac{80}{3} \alpha \beta^{2} \mathrm{TE}_{0 \mathrm{~s} 1}^{\frac{1}{2}} \mathrm{E}_{1 \mathrm{~s} 1}^{\frac{1}{2}} \mathrm{~b}_{5}^{2}+\frac{8}{3}, 16 \alpha^{2} \mathrm{E}_{0 \mathrm{~s} 1} \mathrm{E}_{1 \mathrm{~s} 1} \mathrm{~T}\left(\alpha^{\frac{4}{3}} \mathrm{E}_{0 \mathrm{l} 2}^{\frac{4}{3}}+\frac{4}{3} \alpha \mathrm{E}_{0 \mathrm{~s} 1}^{\frac{1}{2}} \mathrm{E}_{1 \mathrm{~s} 1}^{\frac{1}{2}}+\frac{4}{3}\right)\right\} .
\end{aligned}
$$

Combining equations (4.16), (4.17), and (4.19) together, we obtain

$$
\left\|u_{N}^{n}\right\|_{1}^{2}+\left\|v_{N}^{n}\right\|^{2} \leqslant y_{1}+h \tau \sum_{k=1}^{n-1}\left(\left\|v_{N}^{k}\right\|^{2}+\left\|u_{N}^{k}\right\|_{1}^{2}\right), \quad n=1,2, \cdots, M,
$$

where

$$
\begin{aligned}
y_{1} & =\left(b_{7}+\max \left\{5, c T^{2}\right\}\right)\left(\left\|u_{N}^{0}\right\|_{1}^{2}+\left\|v_{N}^{0}\right\|^{2}+\max _{t}\left(\|\tilde{g}\|_{1, A}^{2}+\|\tilde{f}\|_{1, A}^{2}\right)+\int_{0}^{T}\left\|\tilde{f}_{t}\right\|_{1, A}^{2} d t\right), \\
h & =b_{8}+\max \left\{16 \alpha^{2} E_{0 s 1} E_{1 s 1} T, 20 \beta^{2} b_{5}^{2} T\right\} .
\end{aligned}
$$

Setting $\varphi_{\mathrm{n}}=\left\|\mathfrak{u}_{\mathrm{N}}^{\mathfrak{n}}\right\|_{1}^{2}+\left\|v_{\mathrm{N}}^{\mathfrak{n}}\right\|^{2}$, it is obvious that $\varphi_{1} \leqslant y_{1}$. Then applying Lemma 4.1 to equation (4.20) yields

$$
\varphi_{\mathrm{n}} \leqslant \mathrm{y}_{1} \exp (\mathrm{hT}), \quad \mathrm{n}=1,2, \cdots, M .
$$

Therefore, we obtain the desired result.

\subsection{Convergence of the fully discrete scheme}

In this section, we analyze the convergence of the fully discrete scheme (2.10)-(2.13) by using error estimates method and deduce the order of convergence $\mathrm{O}\left(\tau^{2}+\mathrm{N}^{1-\frac{r}{2}}\right)$.

Now we give the main result of this section.

Theorem 4.6. Assume that the conditions of Lemma 4.2-4.4 hold and $l, l_{t} \in L^{\infty}\left(0, \mathrm{~T} ; \mathrm{H}_{A}^{\mathrm{r}-1}(\mathbf{R})\right), \mathrm{s}, \mathrm{s}_{\mathrm{t}}, \mathrm{s}_{\mathrm{tt}} \in$ $\mathrm{L}^{\infty}\left(0, \mathrm{~T} ; \mathrm{H}_{\mathrm{A}}^{\mathrm{r}}(\mathbf{R})\right), \mathrm{f}, \mathrm{g} \in \mathrm{L}^{\infty}\left(0, \mathrm{~T} ; \mathrm{H}_{\mathrm{A}}^{\mathrm{r}-1}(\mathbf{R})\right), \mathrm{f}_{\mathrm{t}} \in \mathrm{L}^{2}\left(0, \mathrm{~T} ; \mathrm{H}_{\mathrm{A}}^{\mathrm{r}-1}(\mathbf{R})\right)$ for $\mathrm{r} \geqslant 4, \mathrm{l}_{\mathrm{tt}} \in \mathrm{L}^{\infty}\left(0, \mathrm{~T} ; \mathrm{H}^{1}(\mathbf{R})\right), \mathrm{s}_{\mathrm{ttt}} \in$ $\mathrm{L}^{\infty}\left(0, \mathrm{~T} ; \mathrm{H}^{1}(\mathbf{R})\right), \mathrm{l}_{\mathrm{ttt}}, \mathrm{s}_{\mathrm{tttt}} \in \mathrm{L}^{2}\left(0, \mathrm{~T} ; \mathrm{L}^{2}(\mathbf{R})\right)$. Then we have

$$
\left\|s^{n}-s_{N}^{n}\right\|_{1}+\left\|l^{n}-l_{N}^{n}\right\| \leqslant C\left(\tau^{2}+N^{1-\frac{r}{2}}\right), \quad n=0,1,2, \cdots, M,
$$

where $C$ is independent of $\mathrm{N}$ and $\tau$. 
Proof. Let

$$
\left\{\begin{array}{l}
e^{k}=s_{N}^{k}-s^{k}=\left(s_{N}^{k}-P_{N} s^{k}\right)+\left(P_{N} s^{k}-s^{k}\right) \triangleq e_{1}^{k}+e_{2}^{k}, \\
\eta^{k}=l_{N}^{k}-l^{k}=\left(l_{N}^{k}-P_{N} l^{k}\right)+\left(P_{N} l^{k}-l^{k}\right) \triangleq \eta_{1}^{k}+\eta_{2}^{k} .
\end{array}\right.
$$

Using equations (1.1)-(1.4), (2.10)-(2.13), (2.4), and the definition of $\mathrm{P}_{\mathrm{N}}$, we obtain for any $v \in \mathcal{H}_{\mathrm{N}}$, $k=1,2, \cdots, M-1$

$$
\left\{\begin{aligned}
& i\left(e_{1 \hat{\mathrm{t}}}^{k}, v\right)-\left(e_{1 x}^{\bar{k}}, v_{x}\right)=\alpha\left(I_{N}\left(s_{N}^{\bar{k}} l_{N}^{k}\right)-s^{k} l^{k}, v\right)+i\left(s_{t}^{k}-s_{\hat{t}}^{k}, v\right)+\left(\left(P_{N} s^{\bar{k}}\right)_{x}-s_{x}^{k}, v_{x}\right) \quad+\left(I_{N} f^{k}-f^{k}, v\right) \\
&\left(\eta_{1 \hat{t}}^{k}, v\right)+\left(I_{N}\left(\left|s_{N}^{k}\right|^{2}\right)_{x}-\left(\left|s^{k}\right|^{2}\right)_{x}, v\right)=\left(l_{t}^{k}-l_{\hat{\mathrm{t}}}^{k}, v\right)+\left(I_{N} g^{k}-g^{k}, v\right) \\
& e_{1}^{0}=\left(I_{N}-P_{N}\right) s_{0}, \quad \eta_{1}^{0}=\left(I_{N}-P_{N}\right) l_{0} \\
& e_{1}^{1}=s_{N}^{1}-I_{N} s^{1}+\left(I_{N}-P_{N}\right) s^{1}, \eta_{1}^{1}=l_{N}^{1}-I_{N} l^{1}+\left(I_{N}-P_{N}\right) l^{1}
\end{aligned}\right.
$$

We first estimate $\left\|e_{1}^{n}\right\|$. Letting $v=e_{1}^{\bar{k}}$ in equation (4.21) and taking the imaginary part, then using Hölder inequality, we obtain

$$
\begin{aligned}
\frac{1}{2}\left\|e_{1}^{k}\right\|_{\hat{\mathrm{t}}}^{2}= & \alpha \operatorname{Im}\left(\operatorname{I}_{N}\left(s_{N}^{\bar{k}} l_{N}^{k}\right)-s^{k} l^{k}, e_{1}^{\bar{k}}\right)+\operatorname{Re}\left(s_{t}^{k}-s_{\hat{t}}^{k}, e_{1}^{\bar{k}}\right)+\operatorname{Im}\left(\left(P_{N} s^{\bar{k}}\right)_{x}-s_{x}^{k}, v_{x}\right)+\operatorname{Im}\left(I_{N} f^{k}-f^{k}, e_{1}^{\bar{k}}\right) \\
= & \alpha \operatorname{Im}\left(I_{N}\left[s_{N}^{\bar{k}}\left(\eta_{1}^{k}+\eta_{2}^{k}\right)+l^{k} e_{2}^{\bar{k}}\right]+\left(I_{N}-I\right) s^{\bar{k}} l^{k}+l^{k}\left(s^{\bar{k}}-s^{k}\right), e_{1}^{\bar{k}}\right)+\operatorname{Re}\left(s_{t}^{k}-s_{\hat{\mathrm{t}}}^{k}, e_{1}^{\bar{k}}\right) \\
& -\operatorname{Im}\left(e_{2 x x}^{\bar{k}}+\left(s_{x x}^{\bar{k}}-s_{x x}^{k}\right), v\right)+\operatorname{Im}\left(I_{N} f^{k}-f^{k}, e_{1}^{\bar{k}}\right) \\
\leqslant & \alpha\left(\left\|s_{N}^{\bar{k}}\right\|_{\infty}\left(\left\|\eta_{1}^{k}\right\|+\left\|\eta_{2}^{k}\right\|_{N}\right)+\left\|l^{k}\right\|_{\infty}\left\|e_{2}^{\bar{k}}\right\|_{N}+\left\|\left(I_{N}-I\right) s^{\bar{k}} l^{k}\right\|+\left\|l^{k}\right\|\left\|_{\infty}\right\| s^{\bar{k}}-s^{k} \|\right)\left\|e_{1}^{\bar{k}}\right\| \\
& +\left(\left\|s_{t}^{k}-s_{\hat{t}}^{k}\right\|+\left\|e_{2 x x}^{\bar{k}}\right\|+\left\|s_{x x}^{\bar{k}}-s_{x x}^{k}\right\|+\left\|I_{N} f^{k}-f^{k}\right\|\right)\left\|e_{1}^{\bar{k}}\right\| .
\end{aligned}
$$

According to Lemmas 4.2 and 4.3 and the estimates below by using Lemma 2.2, Theorem 2.3, equation (2.6), Taylor's expansion and Hölder inequality

$$
\begin{aligned}
\left\|e_{2}^{\bar{k}}\right\|_{N}+\left\|\eta_{2}^{k}\right\|_{N} & \leqslant c N^{1-\frac{r}{2}}\left(\max _{t}\|s\|_{r-1, A}+\max _{t}\|l\|_{r-1, A}\right),\left\|e_{2 x x}^{\bar{k}}\right\| \leqslant c N^{1-\frac{r}{2}} \max _{t}\|s\|_{r, A} \\
\left\|\left(I_{N}-I\right) s^{\bar{k}} l^{k}\right\| & \leqslant c N^{1-\frac{r}{2}} \max _{t}\|s\|_{r-1, A} \max _{t}\|l\|_{r-1, A},\left\|I_{N} f^{k}-f^{k}\right\| \leqslant c N^{1-\frac{r}{2}} \max _{t}\|f\|_{r-1, A}, \\
\left\|s^{\bar{k}}-s^{k}\right\|_{2} & =\frac{1}{2}\left\|\int_{t_{k-1}}^{t_{k+1}}\left(t_{k+1}-s\right) s_{t t} d s+\int_{t_{k}}^{t_{k-1}}\left(t_{k-1}-s\right) s_{t t} d s\right\|_{2} \\
& \leqslant \frac{\tau^{\frac{3}{2}}}{\sqrt{6}}\left(\int_{t_{k-1}}^{t_{k+1}}\left\|s_{t t}\right\|_{2}^{2} d t\right)^{\frac{1}{2}} \\
& \leqslant \frac{\tau^{2}}{\sqrt{3}} \max _{t}\left\|s_{t t}\right\|_{2}
\end{aligned}
$$

and

$$
\left\|s_{t}^{k}-s_{\hat{t}}^{k}\right\| \leqslant \frac{1}{4 \tau}\left\|\int_{t_{k-1}}^{t_{k+1}}\left(t_{k+1}-s\right)^{2} s_{t t t} d s+\int_{t_{k-1}}^{t_{k}}\left(t_{k-1}-s\right)^{2} s_{t t t} d s\right\| \leqslant \frac{\tau^{2}}{2 \sqrt{5}} \max _{t}\left\|s_{t t t}\right\|,
$$

we obtain

$$
\begin{aligned}
\left\|e_{1}^{k}\right\|_{\hat{t}} \leqslant & \operatorname{cc}_{1} \operatorname{TN}^{1-\frac{r}{2}}\left(\max _{t}\left(\|s\|_{r, A}+\|l\|_{r-1, A}+\|f\|_{r-1, A}\right)+\max _{t}\|s\|_{r-1, A} \max _{t}\|l\|_{r-1, A}\right) \\
& +\frac{c_{1}}{\sqrt{3}} \tau^{2}\left(\max _{t}\left\|s_{t t}\right\|_{2}+\max _{t}\left\|s_{t t t}\right\|\right)+c_{1}\left\|\eta_{1}^{k}\right\|,
\end{aligned}
$$


where $c_{1}=\max \left\{\alpha\|l\|_{L^{\infty}\left((0, T] ; H^{1}(\mathbf{R})\right)}+1, \alpha E_{0 s}^{\frac{1}{2}} E_{1 s}^{\frac{1}{2}}\right\}$. Taking the sum for $k$ from 1 to $n-1$, and using Hölder inequality, we deduce

$$
\left\|e_{1}^{n}\right\|^{2}+\left\|e_{1}^{n-1}\right\|^{2} \leqslant 5\left(\left\|e_{1}^{0}\right\|^{2}+\left\|e_{1}^{1}\right\|^{2}\right)+\frac{40 c_{1}^{2} T^{2}}{3} c_{2} \tau^{4}+c c_{1}^{2} c_{3} T^{2} N^{2-r}+20 c_{1}^{2} T \tau \sum_{k=1}^{n-1}\left\|\eta_{1}^{k}\right\|^{2},
$$

where

$$
c_{2}=\max _{t}\left(\left\|s_{t t}\right\|_{2}^{2}+\left\|s_{t t t}\right\|^{2}\right), c_{3}=\max _{t}\left(\|s\|_{r, A}^{2}+\|l\|_{r-1, A}^{2}+\|f\|_{r-1, A}^{2}\right)+\max _{t}\|s\|_{r-1, A}^{2} \max _{t}\|l\|_{r-1, A}^{2} .
$$

To estimate $\left\|\eta_{1}^{k}\right\|$, letting $v=\eta_{1}^{\bar{k}}$ in equation (4.22), using Hölder inequality, $\mathrm{I}_{\mathrm{N}} u_{\mathrm{N}+1}=\mathrm{P}_{\mathrm{N}} u_{\mathrm{N}+1}$, Lemma 2.2, Theorem 2.3, equation (2.6), and Taylor's expansion lead to

$$
\begin{aligned}
& \left\|\eta_{1}^{k}\right\|_{\hat{\mathrm{t}}} \leqslant \beta\left\|\mathrm{I}_{\mathrm{N}}\left(\left|s_{\mathrm{N}}^{\mathrm{k}}\right|^{2}\right)_{x}-\left(\left|s^{\mathrm{k}}\right|^{2}\right)_{x}\right\|+\left\|l_{\mathrm{t}}^{\mathrm{k}}-\mathrm{l}_{\hat{\mathrm{t}}}^{\mathrm{k}}\right\|+\left\|\left(\mathrm{I}_{\mathrm{N}}-\mathrm{I}\right) \mathrm{g}^{\mathrm{k}}\right\| \\
& \leqslant 2 \beta\left\|I_{N}\left(s_{N}^{k}\left(\bar{s}_{N x}^{k}-\bar{s}_{x}^{k}\right)+\bar{s}_{x}^{k}\left(s_{N}^{k}-s^{k}\right)\right)\right\|+\beta\left\|\left(I_{N}-I\right)\left(\left|s^{k}\right|^{2}\right)_{x}\right\|+\left\|l_{t}^{k}-l_{\hat{t}}^{k}\right\|+\left\|\left(I_{N}-I\right) g^{k}\right\| \\
& \leqslant c_{4}\left(\left\|e_{1}^{k}\right\|_{1}+\left\|e_{2}^{k}\right\|_{1, N}+\left\|\left(I_{N}-I\right)\left(s_{x}^{k} \bar{s}^{k}\right)\right\|\right)+\left\|l_{t}^{k}-l_{\hat{t}}^{k}\right\|+\left\|\left(I_{N}-I\right) g^{k}\right\| \\
& \leqslant \mathrm{cc}_{4} \mathrm{TN}^{\frac{5}{3}-\frac{\mathrm{r}}{2}} \max _{\mathrm{t}}\left(\|s\|_{\mathrm{r}, \mathrm{A}}+\|s\|_{\mathrm{r}, \mathrm{A}}^{2}+\|\mathrm{g}\|_{\mathrm{r}-1, \mathrm{~A}}\right)+\frac{\mathrm{c}_{4} \tau^{\frac{3}{2}}}{2 \sqrt{10}}\left(\int_{\mathrm{t}_{\mathrm{k}-1}}^{\mathrm{t}_{\mathrm{k}+1}}\left\|\mathrm{l}_{\mathrm{ttt}}\right\|^{2} \mathrm{dt}\right)^{\frac{1}{2}}+\mathrm{c}_{4}\left\|e_{1}^{k}\right\|_{1},
\end{aligned}
$$

where $c_{4}=\max \left\{2 \sqrt{2} \beta \mathrm{E}_{0 \mathrm{~s}}^{\frac{1}{2}} \mathrm{E}_{1 s}^{\frac{1}{2}}, 2 \sqrt{2} \beta\|s\|_{\mathrm{L}^{\infty}\left(0, \mathrm{~T} ; \mathrm{H}^{2}(\mathbf{R})\right)}, 2\right\}$. Taking the sum for $\mathrm{k}$ from 1 to $\mathrm{n}-1$, then using Hölder inequality yields

$$
\left\|\eta_{1}^{n}\right\|^{2}+\left\|\eta_{1}^{n-1}\right\|^{2} \leqslant 5\left(\left\|\eta_{1}^{0}\right\|^{2}+\left\|\eta_{1}^{1}\right\|^{2}\right)+c_{4}^{2} c_{5} T \tau^{4}+c c_{4}^{2} c_{6} T^{2} N^{2-r}+20 c_{4}^{2} T \tau \sum_{k=1}^{n-1}\left\|e_{1}^{k}\right\|_{1}^{2}
$$

where $c_{5}=\int_{0}^{T}\left\|l_{t t t}\right\|^{2} d t$, and $c_{6}=c_{3}+\max _{t}\left(\|s\|_{r, A}^{4}+\|g\|_{r-1, A}^{2}\right)$. To evaluate $\left\|e_{1 x}^{k}\right\|$, we let $v=e_{1 \hat{t}}^{k}$ in equation (4.21) and consider the real part, then take the sum for $k$ from 1 to $n-1$, it gives

$$
\begin{aligned}
\left\|e_{1 x}^{n}\right\|^{2}+\left\|e_{1 x}^{n-1}\right\|^{2}= & \left\|e_{1 x}^{0}\right\|^{2}+\left\|e_{1 x}^{1}\right\|^{2}+4 \tau \sum_{k=1}^{n-1} \operatorname{Im}\left(s_{t}^{k}-s_{\hat{\mathrm{t}}}^{k}, e_{1 \hat{\mathrm{t}}}^{k}\right)+4 \tau \sum_{k=1}^{n-1} \operatorname{Re}\left(s_{x}^{k}-\left(P_{N} s^{\bar{k}}\right)_{x}, e_{1 x \hat{\mathrm{t}}}^{k}\right) \\
& +4 \tau \sum_{k=1}^{n-1} \operatorname{Re}\left(\mathrm{I}_{N} f^{k}-f^{k}, e_{1 \hat{\mathrm{t}}}^{\mathrm{k}}\right)-4 \alpha \tau \sum_{\mathrm{k}=1}^{n-1} \operatorname{Re}\left(\mathrm{I}_{N}\left(s_{N}^{\bar{k}} l_{N}^{k}\right)-s^{k} l^{k}, e_{1 \hat{\mathrm{t}}}^{\mathrm{k}}\right) \\
\triangleq & \left\|e_{1 x}^{0}\right\|^{2}+\left\|e_{1 x}^{1}\right\|^{2}+\mathrm{I}_{1}+\mathrm{I}_{2}+\mathrm{I}_{3}+\mathrm{I}_{4} .
\end{aligned}
$$

In view of $\mathrm{I}_{1}-\mathrm{I}_{4}$ on the right hand side of equation (4.27), according to the identity

$$
2 \tau \sum_{k=1}^{n-1}\left(u^{k}, v_{\hat{\mathrm{t}}}^{\mathrm{k}}\right)=-2 \tau \sum_{k=2}^{n-2}\left(u_{\hat{\mathrm{t}}}^{\mathrm{k}}, v^{\mathrm{k}}\right)+\left(\mathrm{u}^{\mathrm{n}-2}, v^{\mathrm{n}-1}\right)+\left(\mathrm{u}^{\mathrm{n}-1}, v^{\mathrm{n}}\right)-\left(\mathrm{u}^{1}, v^{0}\right)-\left(u^{2}, v^{1}\right),
$$

it follows from Hölder inequality and Young's inequality, Lemma 2.2, Taylor's expansion and

$$
\begin{aligned}
\left\|s_{t \hat{t}}^{k}-s_{\hat{t} \hat{t}}^{k}\right\|= & \| \frac{1}{4 \tau}\left(\int_{t_{k}}^{t_{k+1}}\left(t_{k+1}-t\right)^{2} s_{t t t t} d t+\int_{t_{k-1}}^{t_{k}}\left(t_{k-1}-t\right)^{2} s_{t t t t} d t\right) \\
& -\frac{1}{24 \tau^{2}}\left(\int_{t_{k}}^{t_{k+2}}\left(t_{k+2}-t\right)^{3} s_{t t t t} d t+\int_{t_{k-2}}^{t_{k}}\left(t-t_{k-2}\right)^{3} s_{t t t t} d t\right) \| \\
\leqslant & \frac{\tau^{\frac{3}{2}}}{2 \sqrt{10}}\left(\int_{t_{k-1}}^{t_{k+1}}\left\|s_{t t t t}\right\|^{2} d t\right)^{\frac{1}{2}}+\frac{2 \tau^{\frac{3}{2}}}{\sqrt{63}}\left(\int_{t_{k-2}}^{t_{k+2}}\left\|s_{t t t t}\right\|^{2} d t\right)^{\frac{1}{2}}
\end{aligned}
$$


that

$$
\begin{aligned}
\mathrm{I}_{1}= & -4 \tau \operatorname{Im} \sum_{k=2}^{n-2}\left(s_{\mathrm{t} \hat{\mathrm{t}}}^{\mathrm{k}}-s_{\hat{\mathrm{t}} \hat{\mathrm{t}}}^{\mathrm{k}} e_{1}^{\mathrm{k}}\right)+2 \operatorname{Im}\left(\left(s_{\mathrm{t}}^{\mathrm{n}-2}-s_{\hat{\mathrm{t}}}^{\mathrm{n}-2}, e_{1}^{\mathrm{n}-1}\right)+\left(s_{\mathrm{t}}^{\mathrm{n}-1}-s_{\hat{\mathrm{t}}}^{\mathrm{n}-1}, e_{1}^{\mathrm{n}}\right)\right) \\
& -2 \operatorname{Im}\left(\left(s_{\mathrm{t}}^{2}-s_{\hat{\mathrm{t}}}^{2}, e_{1}^{1}\right)-\left(s_{\mathrm{t}}^{1}-s_{\hat{\mathrm{t}}}^{1}, e_{1}^{0}\right)\right) \\
\leqslant & \left\|e_{1}^{0}\right\|^{2}+\left\|e_{1}^{1}\right\|^{2}+\frac{3 \mathrm{~T}}{2}\left(c_{2}+\int_{0}^{T}\left\|s_{\mathrm{ttt} t}\right\|^{2} d \mathrm{t}\right) \tau^{4}+2 \tau \sum_{k=2}^{n-2}\left\|e_{1}^{k}\right\|^{2}+\left(\left\|e_{1}^{\mathfrak{n}}\right\|^{2}+\left\|e_{1}^{n-1}\right\|^{2}\right) .
\end{aligned}
$$

Using Taylor's expansion and Lemma 2.2 and Theorem 2.3, we have

$$
\begin{aligned}
\left\|s_{x \hat{t}}^{k}-s_{x \hat{t}}^{\bar{k}}\right\| & =\left\|\frac{1}{2 \tau} \int_{t_{k-1}}^{t_{k+1}}\left(t_{k+1}-t\right)^{2} s_{x t t t} d t-\frac{1}{4 \tau}\left(\int_{t_{k-1}}^{t_{k+2}}\left(t_{k+2}-t\right)^{2} s_{x t t t} d t+\int_{t_{k-2}}^{t_{k-1}}\left(t_{k-2}-t\right)^{2} s_{x t t t} d t\right)\right\| \\
& \leqslant\left(\frac{93 \tau^{3}}{20}\right)^{\frac{1}{2}}\left(\int_{t_{k-1}}^{t_{k+1}}\left\|s_{x t t t}\right\|^{2} d t+\int_{t_{k-2}}^{t_{k+2}}\left\|s_{x t t t}\right\|^{2} d t\right)^{\frac{1}{2}}, \\
\left\|\left(s_{\hat{t}}^{\bar{k}}-P_{N} s_{\hat{t}}^{\bar{k}}\right)_{x}\right\| & \leqslant c N^{1-\frac{r}{2}}\left\|s_{\hat{\mathfrak{t}}}^{\bar{k}}\right\|_{r-1, A} \leqslant c N^{1-\frac{r}{2}} \max _{t}\left\|s_{t}\right\|_{r, A} .
\end{aligned}
$$

Then using equation (4.28), similarly, we have

$$
\begin{aligned}
\mathrm{I}_{2} \leqslant & \frac{1}{2}\left(\left\|e_{1}^{0}\right\|^{2}+\left\|e_{1 x}^{1}\right\|^{2}\right)+\frac{372 T}{5}\left(c_{2}+\int_{0}^{T}\left\|s_{x t t t}\right\|^{2} d t\right) \tau^{4}+c T\left(c_{3}+\max _{t}\left\|s_{t}\right\|_{r, A}^{2}\right) N^{2-r} \\
& +2 \tau \sum_{k=2}^{n-2}\left\|e_{1 x}^{k}\right\|^{2}+\frac{1}{2}\left(\left\|e_{1 x}^{n}\right\|^{2}+\left\|e_{1 x}^{n-1}\right\|^{2}\right), \\
I_{3} \leqslant & \left\|e_{1}^{0}\right\|^{2}+\left\|e_{1}^{1}\right\|^{2}+c T\left(c_{3}+\int_{0}^{T}\left\|f_{t}\right\|_{r-1, A}^{2} d t\right) N^{2-r}+2 \tau \sum_{k=2}^{n-2}\left\|e_{1 x}^{k}\right\|^{2}+\left\|e_{1}^{n}\right\|^{2}+\left\|e_{1}^{n-1}\right\|^{2} .
\end{aligned}
$$

Rewrite $\mathrm{I}_{4}$ as follows,

$$
\begin{aligned}
I_{4}= & -4 \alpha \tau \operatorname{Re} \sum_{k=1}^{n-1}\left(I_{N}\left(s_{N}^{\bar{k}}\left(\eta_{1}^{k}+\eta_{2}^{k}\right)\right), e_{1 \hat{\mathrm{t}}}^{k}\right)-4 \alpha \tau \operatorname{Re} \sum_{k=1}^{n-1}\left(I_{N}\left(l^{k}\left(e_{1}^{\bar{k}}+e_{2}^{\bar{k}}\right)\right), e_{1 \hat{\mathrm{t}}}^{k}\right) \\
& -4 \alpha \tau \operatorname{Re} \sum_{k=1}^{n-1}\left(\left(I_{N}-I\right)\left(s^{\bar{k}} l^{k}\right), e_{1 \hat{t}}^{k}\right)-4 \alpha \tau \operatorname{Re} \sum_{k=1}^{n-1}\left(l^{k}\left(s^{\bar{k}}-s^{k}\right), e_{1 \hat{t}}^{k}\right) \\
\triangleq & I_{41}+I_{42}+I_{43}+I_{44} .
\end{aligned}
$$

In what follows, we estimate $\mathrm{I}_{41}-\mathrm{I}_{44}$, respectively. Using equation (4.28), and Hölder inequality, we have

$$
\begin{aligned}
& I_{41} \leqslant 4 \alpha \tau \sum_{k=2}^{n-2}\left|\left(I_{N}\left(s_{N}^{\bar{k}+1}\left(\eta_{1 \hat{\mathrm{t}}}^{k}+\eta_{2 \hat{\mathrm{t}}}^{\mathrm{k}}\right)+s_{N \hat{\mathrm{t}}}^{\bar{k}}\left(\eta_{1}^{k-1}+\eta_{2}^{k-1}\right)\right), e_{1}^{k}\right)\right|+2 \alpha\left|\left(s_{N}^{\overline{n-2}} \eta_{1}^{n-2}+s_{N}^{\overline{n-2}} \eta_{2}^{n-2}, e_{1}^{n-1}\right)\right| \\
& +2 \alpha\left|\left(s_{N}^{\bar{n}-1} \eta_{1}^{n-1}+s_{N}^{\overline{n-1}} \eta_{2}^{n-1}, e_{1}^{n}\right)\right|+2 \alpha\left|\left(s_{N}^{\overline{2}} \eta_{1}^{2}+s_{N}^{\overline{2}} \eta_{2}^{2}, e_{1}^{1}\right)\right| \\
& \leqslant 4 \alpha \tau \sum_{k=2}^{n-2}\left(\left\|s_{N}^{\overline{k+1}}\right\|_{\infty}\left(\left\|\eta_{1 \hat{\mathrm{t}}}^{k}\right\|+\eta_{2 \hat{\mathrm{t}}}^{\mathrm{k}} \|_{\mathrm{N}}\right)\left\|e_{1}^{k}\right\|+\left\|e_{1}^{k}\right\|_{\infty}\left\|s_{\mathrm{N} \hat{\mathrm{t}}}^{\bar{k}}\right\|\left(\left\|\eta_{1}^{k-1}\right\|+\left\|\eta_{2}^{k-1}\right\|_{N}\right)\right) \\
& +2 \alpha\left(\left\|s_{N}^{\overline{n-2}}\right\|_{\infty}\left(\left\|\eta_{1}^{n-2}\right\|+\left\|\eta_{2}^{n-2}\right\|_{N}\right)\left\|e_{1}^{n-1}\right\|+\left\|s_{N}^{\overline{n-1}}\right\|_{\infty}\left(\left\|\eta_{1}^{n-1}\right\|+\left\|\eta_{2}^{n-1}\right\|_{N}\right)\left\|e_{1}^{n}\right\|\right. \\
& \left.+\left\|s_{N}^{\overline{2}}\right\|_{\infty}\left(\left\|\eta_{1}^{2}\right\|+\left\|\eta_{2}^{2}\right\|_{N}\right)\left\|e_{1}^{1}\right\|+\left\|s_{N}^{\overline{1}}\right\|_{\infty}\left(\left\|\eta_{1}^{1}\right\|+\left\|\eta_{2}^{1}\right\|_{N}\right)\left\|e_{1}^{0}\right\|\right) \text {. }
\end{aligned}
$$

For the estimate of $\left\|\eta_{1 \hat{\mathrm{t}}}^{\mathrm{k}}\right\|$, using equation (4.22), we find

$$
\left\|\eta_{1 \hat{\mathrm{t}}}^{\mathrm{k}}\right\| \leqslant \mathrm{c}_{4}\left(\left\|e_{1}^{\mathrm{k}}\right\|_{1}+\left\|e_{2}^{\mathrm{k}}\right\|_{1, N}+\left\|\left(\mathrm{I}_{N}-\mathrm{I}\right)\left(\mathrm{s}_{\mathrm{x}}^{\mathrm{k}} \overline{\mathrm{s}}^{\mathrm{k}}\right)\right\|+\left\|\mathrm{l}_{\mathrm{t}}^{\mathrm{k}}-\mathrm{l}_{\hat{\mathrm{t}}}^{\mathrm{k}}\right\|+\left\|\left(\mathrm{I}_{\mathrm{N}}-\mathrm{I}\right) \mathrm{g}^{\mathrm{k}}\right\|\right) \text {. }
$$


Thus we deduce

$$
\begin{aligned}
\mathrm{I}_{41} \leqslant & \mathrm{c}_{7}\left(\left\|e_{1}^{0}\right\|^{2}+\left\|e_{1}^{1}\right\|^{2}+\left\|\eta_{1}^{1}\right\|^{2}\right)+\frac{\mathrm{c}_{2} \mathrm{c}_{7}}{20} \tau^{4}+\mathrm{cc}_{7} \mathrm{~T}\left(\mathrm{c}_{6}+\max _{\mathrm{t}}\left\|\mathrm{l}_{\mathrm{t}}\right\|_{\mathrm{r}-1, \mathrm{~A}}^{2}\right) \mathrm{N}^{2-\mathrm{r}} \\
& +\mathrm{c}_{7} \tau \sum_{\mathrm{k}=1}^{\mathrm{n}-1}\left(\left\|e_{1}^{\mathrm{k}}\right\|_{1}^{2}+\left\|\eta_{1}^{\mathrm{k}}\right\|^{2}\right)+\mathrm{c}_{7}\left(\left\|e_{1}^{\mathrm{n}}\right\|^{2}+\left\|e_{1}^{\mathrm{n}-1}\right\|^{2}+\left\|\eta_{1}^{\mathrm{n}-2}\right\|^{2}+\left\|\eta_{1}^{\mathrm{n}-1}\right\|^{2}+\left\|\eta_{1}^{2}\right\|^{2}\right),
\end{aligned}
$$

where $c_{7}=\sqrt{6} c_{1} c_{4}+E_{0 s}^{\prime}$. Now we estimate $I_{42}$. Applying equation (4.28), Hölder inequality and Young's inequality, we infer that

$$
\begin{aligned}
& I_{42}=2 \tau \sum_{k=2}^{n-2}\left(l_{\hat{\mathrm{t}}}^{k},\left|e_{1}^{k}\right|^{2}\right)_{N}-\alpha\left(\left(l^{n-2},\left|e_{1}^{n-1}\right|^{2}\right)_{N}+\left(l^{n-1},\left|e_{1}^{n}\right|^{2}\right)_{N}-\left(l^{2},\left|e_{1}^{1}\right|^{2}\right)_{N}-\left(l^{1},\left|e_{1}^{0}\right|^{2}\right)_{N}\right) \\
& +4 \tau \operatorname{Re} \sum_{k=2}^{n-2}\left(l_{\hat{\mathrm{t}}}^{k} e_{2}^{\overline{k+1}}+l^{k-1} e_{2 \hat{\mathrm{t}}}^{\bar{k}}, e_{1}^{k}\right)_{N}-2 \alpha \operatorname{Re}\left(\left(l^{n-2} e_{2}^{\overline{n-2}}, e_{1}^{n-1}\right)_{N}-\left(l^{n-1} e_{2}^{\overline{n-1}}, e_{1}^{n}\right)_{N}\right) \\
& +2 \alpha \operatorname{Re}\left(\left(l^{2} e_{2}^{\overline{2}}, e_{1}^{1}\right)_{\mathrm{N}}+\left(l^{2} e_{2}^{\overline{1}}, e_{1}^{0}\right)_{\mathrm{N}}\right) \\
& \leqslant c_{8}\left(\left\|e_{1}^{0}\right\|^{2}+\left\|e_{1}^{1}\right\|^{2}\right)+c c_{8} T\left(c_{3}+\max _{t}\left\|s_{t}\right\|_{r-1, A}^{2}\right) N^{2-r}+c_{8} \tau \sum_{k=2}^{n-2}\left\|e_{1}^{k}\right\|^{2}+c_{8}\left(\left\|e_{1}^{n}\right\|^{2}+\left\|e_{1}^{n-1}\right\|^{2}\right),
\end{aligned}
$$

where $c_{8}=4\left(\left\|l_{t}\right\|_{\mathrm{L}^{\infty}\left(0, \mathrm{~T} ; \mathrm{L}^{2}(\mathbf{R})\right)}^{\frac{1}{2}}\left\|\mathrm{l}_{x \mathrm{t}}\right\|_{\mathrm{L}^{\infty}\left(0, \mathrm{~T} ; \mathrm{L}^{2}(\mathbf{R})\right)}^{\frac{1}{2}}+\|\mathrm{l}\|_{\mathrm{L}^{\infty}\left(0, \mathrm{~T} ; \mathrm{L}^{\infty}(\mathbf{R})\right)}\right)$. Similarly, we obtain

$$
\begin{aligned}
\mathrm{I}_{43} \leqslant & \left\|e_{1}^{0}\right\|^{2}+\left\|e_{1}^{1}\right\|^{2}+c\left(c_{3}+\max _{t}\|s\|_{r-1, A}^{2} \int_{0}^{T}\left\|l_{t}\right\|_{r-1, A}^{2} d t+\max _{t}\|l\|_{r-1, A}^{2} \int_{0}^{T}\left\|s_{t}\right\|_{r-1, A}^{2} d t\right) N^{2-r} \\
& +2 \tau \sum_{k=2}^{n-2}\left\|e_{1}^{k}\right\|^{2}+\left\|e_{1}^{\mathfrak{n}}\right\|^{2}+\left\|e_{1}^{n-1}\right\|^{2} \\
I_{44} \leqslant & c_{8}\left(\left\|e_{1}^{1}\right\|^{2}+\left\|e_{1}^{0}\right\|^{2}\right)+\frac{186}{5} c_{2} c_{8} \tau^{4}+c_{8} \tau \sum_{k=2}^{n-2}\left\|e_{1}^{k}\right\|^{2}+c_{8}\left(\left\|e_{1}^{\mathfrak{n}}\right\|^{2}+\left\|e_{1}^{n-1}\right\|^{2}\right) .
\end{aligned}
$$

Substituting $\mathrm{I}_{41}-\mathrm{I}_{44}$ into equation (4.29) leads to

$$
\begin{aligned}
\mathrm{I}_{4} \leqslant & \mathrm{c}_{9}\left(\left\|e_{1}^{0}\right\|^{2}+\left\|e_{1}^{1}\right\|^{2}+\left\|\eta_{1}^{1}\right\|^{2}\right)+\frac{186}{5} c_{2}\left(c_{7}+c_{8}\right) c_{9} T \tau^{4}+c_{9} c_{10} T^{2} N^{2-r} \\
& +c_{9} \tau \sum_{k=1}^{n-1}\left(\left\|e_{1}^{k}\right\|_{1}^{2}+\left\|\eta_{1}^{k}\right\|^{2}\right)+c_{9}\left(\left\|e_{1}^{n}\right\|^{2}+\left\|e_{1}^{n-1}\right\|^{2}+\left\|\eta_{1}^{n-2}\right\|^{2}+\left\|\eta_{1}^{n-1}\right\|^{2}+\left\|\eta_{1}^{2}\right\|^{2}\right)
\end{aligned}
$$

where $c_{9}=c_{7}+2 c_{8}+2$ and

$$
c_{10}=c_{6}+\max _{t}\left(\left\|s_{t}\right\|_{r, A}^{2}+\left\|l_{t}\right\|_{r-1, A}^{2}\right)+\max _{t}\|s\|_{r-1, A}^{2} \int_{0}^{T}\left\|l_{t}\right\|_{r-1, A}^{2} d t+\max _{t}\|l\|_{r-1, A}^{2} \int_{0}^{T}\left\|s_{t}\right\|_{r-1, A}^{2} d t .
$$

Substituting $\mathrm{I}_{1}-\mathrm{I}_{4}$ into equation (4.27) and using equations (4.25) and (4.26), we obtain

$$
\begin{aligned}
\left\|e_{1}^{\mathfrak{n}}\right\|_{1}^{2}+\left\|\eta_{1}^{\mathfrak{n}}\right\|^{2} \leqslant & \left(7 c_{9}+20\right)\left(\left\|e_{1}^{0}\right\|_{1}^{2}+\left\|e_{1}^{1}\right\|_{1}^{2}+\left\|\eta_{1}^{0}\right\|^{2}+\left\|\eta_{1}^{1}\right\|^{2}\right)+c_{11}\left(\tau^{4}+N^{2-r}\right) \\
& +c_{12} \tau \sum_{k=1}^{n-1}\left(\left\|e_{1}^{k}\right\|_{1}^{2}+\left\|\eta_{1}^{k}\right\|^{2}\right)
\end{aligned}
$$

where $c_{12}=2 c_{9}+4+20\left(c_{9}+3\right) T \max \left\{c_{1}^{2}, c_{4}^{2}\right\}$ and

$$
c_{11}=T^{2} \max \left\{( 7 5 ( c _ { 7 } + c _ { 8 } ) c _ { 9 } + 7 6 + ( c _ { 9 } + 2 ) ( \frac { 4 0 c _ { 1 } ^ { 2 } } { 3 } + c _ { 4 } ^ { 2 } ) ) \left(c_{2}+c_{5}\right.\right.
$$




$$
\left.\left.+\int_{0}^{T}\left(\left\|s_{x t t t}\right\|^{2}+\left\|s_{t t t t}\right\|^{2}\right) d t\right), c\left(c_{9}+\left(c_{9}+3\right)\left(c_{1}^{2}+c_{2}^{2}\right)\right)\left(c_{10}+\int_{0}^{T}\left\|f_{t}\right\|_{r-1, A}^{2} d t\right)\right\} .
$$

Now we consider the initial values, it follows from equations (4.23), (4.24), and Lemma 2.2, Theorem 2.3, equation (2.6) and Hölder inequality, we derive that

$$
\begin{aligned}
\left\|e_{1}^{0}\right\|_{1}^{2} & \leqslant c N^{2-r}\left\|s_{0}\right\|_{r, A}^{2} \cdot\left\|\eta_{1}^{0}\right\| \leqslant c N^{2-r}\left\|l_{0}\right\|_{r-1, A}^{2} \\
\left\|e_{1}^{1}\right\|_{1}^{2} & \leqslant 2\left\|I_{N} \int_{0}^{\tau}(\tau-t) s_{t t} d t\right\|_{1}^{2}+2\left\|\left(I_{N}-P_{N}\right) s^{1}\right\|_{1}^{2} \\
& \leqslant 4\left\|\left(I_{N}-I\right) \int_{0}^{\tau}(\tau-t) s_{t t} d t\right\|_{1}^{2}+4\left\|\int_{0}^{\tau}(\tau-t) s_{t t} d t\right\|_{1}^{2}+2\left\|\left(I_{N}-P_{N}\right) s^{1}\right\|_{1}^{2} \\
& \leqslant c N^{2-r} \max _{t}\left(\|s\|_{r, A}^{2}+\left\|s_{t t}\right\|_{r, A}^{2}\right)+\frac{4}{3} \tau^{4} \max _{t}\left\|s_{t t}\right\|_{1}^{2} .
\end{aligned}
$$

Similarly, using Lemma 3.1 given in [9] where $\|v\|_{N} \leqslant c\left(\|v\|+\mathrm{cN}^{-\frac{1}{6}}|v|_{1}\right)$, we have

$$
\left\|\eta_{1}^{1}\right\|^{2} \leqslant 2\left\|I_{N} \int_{0}^{\tau}(\tau-t) l_{t t} d t\right\|^{2}+2\left\|\left(I_{N}-P_{N}\right) l^{1}\right\|^{2} \leqslant c N^{2-r} \max _{t}\|l\|_{r-1, A}^{2}+c \tau^{4} \max _{t}\left\|l_{t t}\right\|^{2} .
$$

Thus we have

$$
\left\|e_{1}^{\mathfrak{n}}\right\|_{1}^{2}+\left\|\eta_{1}^{n}\right\|^{2} \leqslant c_{13}\left(N^{2-r}+\tau^{4}\right)+c_{12} \tau \sum_{k=1}^{n-1}\left(\left\|e_{1}^{k}\right\|_{1}^{2}+\left\|\eta_{1}^{k}\right\|^{2}\right),
$$

where $c_{13}=c_{11}+c c_{3}+c \max _{t}\left(\left\|s_{t t}\right\|_{r, A}^{2}+\left\|s_{t t}\right\|_{1}^{2}+\left\|l_{t t}\right\|_{1}^{2}\right)$. Applying Lemma 4.1 for equation (4.30), we deduce that

$$
\left\|e_{1}^{\mathfrak{n}}\right\|_{1}^{2}+\left\|\eta_{1}^{\mathfrak{n}}\right\|^{2} \leqslant c_{13}\left(\tau^{4}+N^{2-r}\right) \exp \left(c_{12} T\right), \quad n=1,2, \cdots, M
$$

Finally, using the triangle inequality and Lemma 2.2 , we have

$$
\left\|s^{n}-s_{N}^{n}\right\|_{1}+\left\|l^{n}-l_{N}^{n}\right\| \leqslant\left\|e_{2}^{n}\right\|_{1}+\left\|e_{1}^{n}\right\|_{1}+\left\|\eta_{2}^{n}\right\|+\left\|\eta_{1}^{n}\right\| \leqslant C\left(\tau^{2}+N^{1-\frac{r}{2}}\right), \quad n=0,1, \cdots, M,
$$

where $C=\sqrt{2 c_{13}} \exp \left(c_{12} T / 2\right)$.

Consequently, we finish the proof of this theorem.

\section{Numerical results}

In this section, we describe the numerical implementations and present the numerical result for the fully discrete scheme of Hermite pseudospectral method and modified Hermite spectral method, respectively.

We consider LS equations (1.1)-(1.4) with $\alpha=\beta=1$ and the following source terms:

$$
f(x, t)=-\frac{\left(\cosh ^{2}(x+2 t)+3\right) e^{i(t-x)}}{\cosh ^{3}(x+2 t)} \text { and } g(x, t)=-\frac{6 \sinh ^{2}(x+2 t)}{\cosh ^{3}(x+2 t)} .
$$

The exact solutions of this example are:

$$
s(x, t)=\operatorname{sech}(x+2 t) e^{i(t-x)} \text { and } l(x, t)=\operatorname{sech}^{2}(x+2 t) .
$$

For the Hermite pseudospectral method, we choose Lagrange basis functions $\ell_{m}(x)$ with weight $\omega_{m}(x)=$ $e^{-\frac{x^{2}}{2}} / e^{-\frac{x_{m}^{2}}{2}}$ and denote $h_{m}(x)=\ell_{m}(x) \omega_{m}(x)$, then we rewrite the numerical solutions as

$$
s_{N}^{k+1}(x)=\sum_{m=0}^{N} s_{m}^{k+1} h_{m}(x), \quad l_{N}^{k+1}(x)=\sum_{n=0}^{N} l_{n}^{k+1} h_{n}(x),
$$


where $s_{m}^{k+1}=s_{N}^{k+1}\left(x_{m}\right)$ and $l_{n}^{k+1}=l_{N}^{k+1}\left(x_{n}\right)$ are the nodal values of discrete solutions. Then we obtain the following system of linear algebraic equations:

$$
\begin{aligned}
\left(i A_{1}-\frac{\tau}{2} B_{1}-\frac{\tau}{2} C_{1}\right) s_{1}^{k+1} & =\left(i A_{1}+\frac{\tau}{2} B_{1}+\frac{\tau}{2} C_{1}\right) s_{1}^{k}+\tau A_{1} f^{k+\frac{1}{2}}, \\
l_{1}^{k+1} & =l_{1}^{k}-2 \tau \operatorname{ReD}_{1} s_{1}^{k}+\tau g^{k+\frac{1}{2}}
\end{aligned}
$$

where

$$
\begin{aligned}
s_{1}^{k+1} & =\left(s_{0}^{k+1}, s_{1}^{k+1}, \cdots, s_{N}^{k+1}\right)^{\top}, & l_{1}^{k+1} & =\left(l_{0}^{k+1}, l_{1}^{k+1}, \cdots, l_{N}^{k+1}\right)^{\top}, \\
f^{k} & =\left(f^{k+\frac{1}{2}}\left(x_{0}\right), f^{k+\frac{1}{2}}\left(x_{1}\right), \cdots, f^{k+\frac{1}{2}}\left(x_{N}\right)\right)^{\top}, & g^{k} & =\left(g^{k+\frac{1}{2}}\left(x_{0}\right), g^{k+\frac{1}{2}}\left(x_{1}\right), \cdots, g^{k+\frac{1}{2}}\left(x_{N}\right)\right)^{\top}, \\
A_{1} & =\left(a_{i j}\right)_{i, j=0,1, \cdots, N}, & a_{i j} & =w_{j} \delta_{i j}, \quad B_{1}=H^{\top} A_{1} H,
\end{aligned}
$$

$\mathrm{H}$ is the first-order Hermite differential matrix of Hermite functions relative to $\left\{x_{j}\right\}_{j=0}^{N}$, and

$$
\mathrm{C}_{1}=\operatorname{diag}\left(\mathrm{l}_{0}^{\mathrm{k}} w_{0}, \mathrm{l}_{1}^{\mathrm{k}} w_{1}, \cdots, \mathrm{l}_{\mathrm{N}}^{\mathrm{k}} w_{\mathrm{N}}\right), \mathrm{D}_{1}=\operatorname{diag}\left(\overline{\boldsymbol{s}^{\mathrm{k}}}\right) \mathrm{H}
$$

For the modified Hermite spectral method, we rewrite the numerical solutions as follows,

$$
s_{N}^{k+1}(x)=\sum_{m=0}^{N} \hat{s}_{m}^{k+1} \hat{H}_{m}(x), \quad l_{N}^{k+1}(x)=\sum_{n=0}^{N} \hat{l}_{n}^{k+1} \hat{H}_{n}(x),
$$

where $\hat{s}_{m}^{k+1}$ and $\hat{l}_{\mathfrak{n}}^{k+1}$ are Hermite coefficients. Then we obtain the following system of linear algebraic equations:

$$
\left(\mathrm{iI}-\tau \mathrm{A}_{2}-\tau \mathrm{B}_{2}\right) \boldsymbol{s}_{2}^{\mathrm{k}+1}=\left(\mathrm{iI}+\tau \mathrm{A}_{2}+\tau \mathrm{B}_{2}\right) \boldsymbol{s}_{2}^{\mathrm{k}-1}+2 \tau \phi^{\mathrm{k}}, \quad \mathrm{I} l_{2}^{\mathrm{k}+1}=\mathrm{I} l_{2}^{\mathrm{k}-1}-4 \tau \operatorname{ReC}_{2} s_{2}^{\mathrm{k}}+2 \tau \psi^{\mathrm{k}},
$$

where $s_{2}^{k+1}=\left(\hat{s}_{0}^{k+1}, \hat{s}_{1}^{k+1}, \ldots, \hat{s}_{N}^{k+1}\right)^{\top}$ and $l_{2}^{k+1}=\left(\hat{l}_{0}^{k+1}, \hat{l}_{1}^{k+1}, \ldots, \hat{l}_{N}^{k+1}\right)^{\top}$. According to equations (2.2) and (2.3), we know that $I$ is the identity matrix, $A_{2}$ is a pentadiagonal matrix, $B_{2}=D_{2} E_{1} D_{2}^{T}$ is a symmetrical matrix, $\mathrm{C}_{2}=\mathrm{D}_{2} \mathrm{E}_{2} \mathrm{D}_{2}^{(1)}, \boldsymbol{\phi}^{\mathrm{k}}=\mathrm{D}_{2} \mathrm{E}_{3}$, and $\psi^{\mathrm{k}}=\mathrm{D}_{2} \mathrm{E}_{4}$, where

$$
\begin{aligned}
& \mathrm{D}_{2}=\left(\mathrm{d}_{\mathrm{ij}}\right)=\left(\hat{\mathrm{H}}_{\mathrm{i}}\left(\mathrm{x}_{\mathbf{j}}\right)\right) \text {, } \\
& E_{1}=\operatorname{diag}\left(l_{N}^{k}\left(x_{0}\right) w_{0}, \cdots, l_{N}^{k}\left(x_{N}\right) w_{N}\right) \text {, } \\
& D_{2}^{(1)}=\left(d_{i j}^{\prime}\right)=\left(\hat{H}_{i x}\left(x_{j}\right)\right), \quad i, j=0,1, \cdots, N, \\
& E_{2}=\operatorname{diag}\left(\bar{s}_{N}^{k}\left(x_{0}\right) w_{0}, \cdots, \bar{s}_{N}^{k}\left(x_{N}\right) w_{N}\right) \text {, }
\end{aligned}
$$

and

$$
E_{3}=\left(f^{k}\left(x_{0}\right) w_{0}, \cdots, f^{k}\left(x_{N}\right) w_{N}\right)^{T}, \quad E_{4}=\left(g^{k}\left(x_{0}\right) w_{0}, \cdots, g^{k}\left(x_{N}\right) w_{N}\right)^{T} .
$$

To see the order of the accuracy for Hermite pseudospectral method and modified Hermite spectral method, we present Tables 1 and 2.

From Tables 1 and 2, it is clear that both $\mathrm{L}^{2}$-error and $\mathrm{L}^{\infty}$-error with different $\tau$ for given $\mathrm{N}$ indicate a one-order accuracy in time for Hermite pseudospectral method and second-order accuracy in time for modified Hermite spectral method.

The errors with different $N$ for given $\tau$, the accuracy for modified Hermite spectral method is still higher than Hermite pseudospectral method. We see that the accuracy reaches e-06 at $\mathrm{N}=128$ when $\tau=10^{-3}$ in Table 2, while it does not achieve the same accuracy when $\mathrm{N}$ is no more than 64 . The main reason is that $C N^{1-\frac{r}{2}}$ in the convergence analysis result $C\left(\tau^{2}+N^{1-\frac{r}{2}}\right)$ occupies the leading role. We have to point out that the coefficient $C$ which includes $\|\cdot\|_{r, A}$ really affects the accuracy. As is known that the norm $\|\cdot\|_{r, A}$ becomes bigger as $r$ increases. We take $N=64$ for example, if $N^{1-\frac{r}{2}}$ is the same accuracy as e-06, we need $r>8$, while $\|s\|_{8, A}=1.5314 \mathrm{e}+05$, then $\|s\|_{8, \mathrm{~A}} \mathrm{~N}^{-3}$ has the accuracy e-01 which is much bigger than the accuracy e-05 in Table 2.

Thus the data in Tables 1 and 2 confirm our theoretical analysis. 
Table 1: Hermite pseudospectral method for LS equations

\begin{tabular}{|c|c|c|c|c|c|}
\hline \multirow{2}{*}{$\tau$} & \multirow{2}{*}{$\mathrm{N}$} & \multicolumn{2}{|c|}{$e$} & \multicolumn{2}{|c|}{$\eta$} \\
\hline & & $\mathrm{L}^{2}$-error & $\mathrm{L}^{\infty}$-error & $\mathrm{L}^{2}$-error & $\mathrm{L}^{\infty}$-error \\
\hline $10^{-}$ & \multirow{3}{*}{128} & $2.7606 \mathrm{e}-02$ & $1.4134 \mathrm{e}-02$ & $6.4815 \mathrm{e}-02$ & $5.1296-02$ \\
\hline $10^{-2}$ & & $2.4977 \mathrm{e}-03$ & $1.1822 \mathrm{e}-03$ & $6.5258 \mathrm{e}-03$ & $5.1278 \mathrm{e}-03$ \\
\hline $10^{-3}$ & & $2.4786 \mathrm{e}-04$ & $1.1657 \mathrm{e}-04$ & $6.5342 \mathrm{e}-04$ & $5.1295 \mathrm{e}-04$ \\
\hline \multirow{5}{*}{$10^{-4}$} & $\overline{8}$ & 1.4534e-01 & 8.7838e-02 & $4.7363 \mathrm{e}-02$ & $\overline{4.2161 \mathrm{e}-02}$ \\
\hline & 16 & $3.0897 \mathrm{e}-02$ & $2.2134 \mathrm{e}-02$ & $8.9822 \mathrm{e}-03$ & $7.2374 \mathrm{e}-03$ \\
\hline & 32 & $2.7714 \mathrm{e}-03$ & $2.1416 \mathrm{e}-03$ & $5.9142 \mathrm{e}-04$ & $5.3013 \mathrm{e}-04$ \\
\hline & 64 & $1.0633 \mathrm{e}-04$ & $8.6102 \mathrm{e}-05$ & $6.6901 \mathrm{e}-05$ & $6.0466 \mathrm{e}-05$ \\
\hline & 128 & $2.4794 \mathrm{e}-05$ & $1.1692 \mathrm{e}-05$ & $6.5351 \mathrm{e}-05$ & $5.1260 \mathrm{e}-05$ \\
\hline
\end{tabular}

Table 2: Modified Hermite spectral method for LS equations

\begin{tabular}{|c|c|c|c|c|c|}
\hline \multirow{2}{*}{$\tau$} & \multirow{2}{*}{$\mathrm{N}$} & \multicolumn{2}{|c|}{ e } & \multicolumn{2}{|c|}{$\eta$} \\
\hline & & $\mathrm{L}^{2}$-error & $\mathrm{L}^{\infty}$-error & $\mathrm{L}^{2}$-error & $\mathrm{L}^{\infty}$-error \\
\hline $10^{-}$ & \multirow{3}{*}{128} & $5.2126 \mathrm{e}-02$ & $2.3683 \mathrm{e}-02$ & $3.7789 \mathrm{e}-02$ & $1.5177 \mathrm{e}-02$ \\
\hline $10^{-2}$ & & $4.7371 \mathrm{e}-04$ & $1.9027 \mathrm{e}-04$ & $2.8829 \mathrm{e}-04$ & $1.1614 \mathrm{e}-04$ \\
\hline $10^{-3}$ & & $4.7508 \mathrm{e}-06$ & $1.9052 \mathrm{e}-06$ & $2.8605 \mathrm{e}-06$ & $1.1781 \mathrm{e}-06$ \\
\hline \multirow{5}{*}{$10^{-3}$} & $\overline{16}$ & $\overline{77.0108 \mathrm{e}-03}$ & 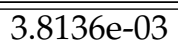 &  & $\overline{\overline{7} 7.7590 \mathrm{e}-03}$ \\
\hline & 32 & $1.2201 \mathrm{e}-03$ & $1.0893 \mathrm{e}-03$ & $1.1101 \mathrm{e}-03$ & $5.4457 \mathrm{e}-04$ \\
\hline & 64 & $5.3261 \mathrm{e}-05$ & $4.8205 e-05$ & $3.2772 \mathrm{e}-05$ & $1.4331 \mathrm{e}-05$ \\
\hline & 128 & $4.7508 \mathrm{e}-06$ & $1.9052 \mathrm{e}-06$ & $2.8605 e-06$ & $1.1781 \mathrm{e}-06$ \\
\hline & 256 & $5.6344 \mathrm{e}-06$ & $1.9431 \mathrm{e}-06$ & $3.3940 \mathrm{e}-06$ & $1.1345 \mathrm{e}-06$ \\
\hline
\end{tabular}

\section{Conclusions}

We prove the convergence of the Hermite pseudospectral method and study the modified Hermite spectral method including a priori estimates, unconditional numerical stability and the convergence of the fully discrete scheme. Numerical results also verify our theoretical analysis. The reason that we choose forward difference scheme in time for pseudospectral method is because of difference quotient in equation (3.15), if we select central difference scheme, then the first level difference quotient will not satisfy the accuracy we need, thus we have to adopt the scheme (2.7)-(2.9) we give.

It should be pointed out that the conditions of exact solutions $s, l$ in our assumptions (Theorems 3.3, 4.6) may be restrictive. In the future, we will try to prove the existence of the solutions in weighted Sobolev space. As for spectral method, one of the most interesting problems related to the partial differential equations is how to establish an estimate with respect to the data regularity.

\section{Acknowledgment}

The authors were supported by the NSF of China (Nos. 11272024, 11672011).

\section{References}

[1] J. Aguirre, J. Rivas, Hermite pseudospectral approximations, An error estimate, J. Math. Anal. Appl., 304 (2005), 189-197. 2

[2] J. Aguirre, J. Rivas, Spectral methods based on Hermite functions for linear hyperbolic equations, Numer. Methods Partial Differential Equations, 28 (2012), 1696-1716. 1

[3] G. Akrivis, D. T. Papageorgiou, Y.-S. Smyrlis, Computational study of the dispersively modified Kuramoto-Sivashinsky equation, SIAM J. Sci. Comput., 34 (2012), A792-A813. 1

[4] G. A. Baker, V. A. Dougalis, O. A. Karakashian, Convergence of Galerkin approximations for the Korteweg-de Vries equation, Math. Comp., 40 (1983), 419-433. 1

[5] J. P. Boyd, Chebyshev and Fourier spectral methods, Second edition, Dover Publications, Inc., Mineola, NY, (2001). 1 
[6] O. Coulaud, D. Funaro, O. Kavian, Laguerre spectral approximation of elliptic problems in exterior domains, Spectral and high order methods for partial differential equations, Como, (1989), Comput. Methods Appl. Mech. Engrg., 80 (1990), 451-458. 1

[7] B.-Y. Guo, Error estimation of Hermite spectral method for nonlinear partial differential equations, Math. Comp., 68 (1999), 1067-1078. 1

[8] B.-Y. Guo, J. Shen, Laguerre-Galerkin method for nonlinear partial differential equations on a semi-infinite interval, Numer. Math., 86 (2000), 635-654. 1

[9] B.-Y. Guo, J. Shen, C.-L. Xu, Spectral and pseudospectral approximations using Hermite functions: application to the Dirac equation, Challenges in computational mathematics, Pohang, (2001), Adv. Comput. Math., 19 (2003), 35-55. 2, 4.3

[10] B.-Y. Guo, L.-L. Wang, Modified Laguerre pseudospectral method refined by multidomain Legendre pseudospectral approximation, J. Comput. Appl. Math., 190 (2006), 304-324. 1

[11] B.-Y. Guo, C.-L. Xu, Hermite pseudospectral method for nonlinear partial differential equations, M2AN Math. Model. Numer. Anal., 34 (2000), 859-872. 1

[12] B.-Y. Guo, X.-Y. Zhang, A new generalized Laguerre spectral approximation and its applications, J. Comput. Appl. Math., 184 (2005), 382-403. 1

[13] H.-P. Ma, W.-W. Sun, Optimal error estimates of the Legendre-Petrov-Galerkin method for the Korteweg-de Vries equation, SIAM J. Numer. Anal., 39 (2001), 1380-1394. 3.2

[14] H.-P. Ma, W.-W. Sun, T. Tang, Hermite spectral methods with a time-dependent scaling for parabolic equations in unbounded domains, SIAM J. Numer. Anal., 43 (2005), 58-75. 1

[15] H.-P. Ma, T.-G. Zhao, A stabilized Hermite spectral method for second-order differential equations in unbounded domains, Numer. Methods Partial Differential Equations, 23 (2007), 968-983. 1

[16] B. Pelloni, V. A. Dougalis, Error estimates for a fully discrete spectral scheme for a class of nonlinear, nonlocal dispersive wave equations, Appl. Numer. Math., 37 (2001), 95-107. 1

[17] A. Quarteroni, A. Valli, Numerical approximation of partial differential equations, Springer Series in Computational Mathematics, Springer-Verlag, Berlin, (2008). 4.1

[18] J. Shen, Stable and efficient spectral methods in unbounded domains using Laguerre functions, SIAM J. Numer. Anal., 38 (2000), 1113-1133. 1

[19] J. Shen, T. Tang, L.-L. Wang, Spectral methods. Algorithms, analysis and applications, Springer Series in Computational Mathematics, Springer, Heidelberg, (2011). 2

[20] J. Shen, L.-L. Wang, Laguerre and composite Legendre-Laguerre dual-Petrov-Galerkin methods for third-order equations, Discrete Contin. Dyn. Syst. Ser. B, 6 (2006), 1381-1402. 1

[21] G. Szegö, Orthogonal Polynomials, Amer. Math. Soc. Colloq. Publ., Amer. Math. Soc., Providence, RI, (1939). 2

[22] T. Tang, The Hermite spectral method for Gaussian-type functions, SIAM J. Sci. Comput., 14 (1993), 594-606. 1

[23] X.-M. Xiang, Z.-Q. Wang, Generalized Hermite spectral method and its applications to problems in unbounded domains, SIAM J. Numer. Anal., 48 (2010), 1231-1253. 2.1, 2.2

[24] X.-M. Xiang, Z.-Q. Wang, Generalized Hermite approximations and spectral method for partial differential equations in multiple dimensions, J. Sci. Comput., 57 (2013), 229-253. 1 